\title{
Spectrum of anisotropic exponents in hydrodynamic systems with pressure
}

\author{
Itai Arad and Itamar Procaccia \\ Deptartment of Chemical Physics, The Weizmann Institute of Science, Rehovot 76100, Israel
}

(version of December 29, 2000)

\begin{abstract}
We discuss the scaling exponents characterizing the power-law behavior of the anisotropic components of correlation functions in turbulent systems with pressure. The anisotropic components are conveniently labeled by the angular momentum index $\ell$ of the irreducible representation of the $\mathrm{SO}(3)$ symmetry group. Such exponents govern the rate of decay of anisotropy with decreasing scales. It is a fundamental question whether they ever increase as $\ell$ increases, or they are bounded from above. The equations of motion in systems with pressure contain nonlocal integrals over all space. One could argue that the requirement of convergence of these integrals bounds the exponents from above. It is shown here on the basis of a solvable model (the "linear pressure model"), that this is not necessarily the case. The model introduced here is of a passive vector advection by a rapidly varying velocity field. The advected vector field is divergent free and the equation contains a pressure term that maintains this condition. The zero modes of the second-order correlation function are found in all the sectors of the symmetry group. We show that the spectrum of scaling exponents can increase with $\ell$ without bounds, while preserving finite integrals. The conclusion is that contributions from higher and higher anisotropic sectors can disappear faster and faster upon decreasing the scales also in systems with pressure.
\end{abstract}

\section{INTRODUCTION}

Turbulent flows are often forced in an anisotropic fashion. The anisotropy has a significant effect on a variety of measured turbulence characteristics. We are interested in the effect of anisotropy on statistical quantities, especially the low-order structure functions of velocity differences across a scale $R$. In perfectly isotropic systems such objects are expected to display pure scaling behavior in the limit of high Reynolds number. We have suggested recently [1] that in the presence of anisotropy, the structure functions are no longer pure power laws. Instead, components of the structure functions that belong to different irreducible representations (sectors) of the $\mathrm{SO}(3)$ group possess different scaling exponents. Each of these sectors is characterized by the angular momentum indices $\ell$ and $m$. By projecting the structure function onto the different sectors, we could measure [2, 4 ] the universal scaling exponents in each sector separately.

The spectrum of anisotropic exponents is particularly accessible in linear problems like passive scalar [5] and passively advected magnetic fields [6]. In both the models, the equations of motion are isotropic and as a result the existence of universal anisotropic exponents can be proven [7]8]. Additionally the isotropy of the equations implies that the scaling exponents depend on $\ell$ but not on $m$. One of the important results of the analysis is that the discrete spectrum of anisotropic exponents is strictly increasing as a function of $\ell$. This explains the isotropization of the statistics as smaller and smaller scales are observed. Since the scaling exponents $\zeta$ appear in power laws of the type $(R / L)^{\zeta}$, with $L$ being some typical outer scale and $R \ll L$, the larger is the exponent, the faster is the decay of the contribution as the scale $R$ diminishes. Therefore the gap between the leading, isotropic exponent, and the next available exponent governs the rate of isotropization.

Experiments and simulations on Navier-Stokes (NS) turbulence also indicate that anisotropic sectors possess larger scaling exponents than the isotropic sector 24. However, to date, the exponents for $\ell>2$ were not determined with sufficient accuracy. We thus do not know whether the higher sectors are characterized by an ever increasing exponents, or whether the exponents saturate. This issue is theoretically puzzling because of the effects of pressure. The inversion of the pressure in terms of the Green's function of the Laplacian operator introduces integrals over the domain of turbulence. These integrals manifest the nonlocality of the problem, and are present in both the dynamical equation, and in the equations for the correlation functions. When considering a spatially homogeneous turbulence, the turbulent domain is usually taken to be infinite. The physical boundary conditions at scale $L$, are mimicked by employing a homogeneous forcing that acts at that scale. In this case, the integrals that result from the pressure term are over all $\mathbb{R}^{3}$, and their convergence has to be guaranteed. The question that we want to address in this paper is the following: does the requirement of the convergence of the integrals necessarily bound the spectrum of the scaling exponents from above? Since the correlation functions appear in the integrand, an unbounded spectrum implies a rapidly increasing integrand as a function of the length scale. On the face of it, at some point the integrals must diverge in the infrared. It would appear therefore that either there must be a limit to the magnitude of the scaling exponents, or that the integrals converge due to an infrared crossover in the correlation functions. The latter scenario looks physically reasonable, yet in the presence of pressure integrals, seems to break scale invariance in the inertial range. To demonstrate that, consider a typical integral term of the form, 


$$
\int d \boldsymbol{y} G(\boldsymbol{r}-\boldsymbol{y}) C(\boldsymbol{y})
$$

Here $G(\boldsymbol{r})=-1 /(4 \pi|\boldsymbol{r}|)$ is the infinite domain Green function of the Laplacian operator, and $C(\boldsymbol{r})$ is some statistical object, which is expected to be scale invariant in the inertial range. If $C(\boldsymbol{r})$ has an infrared crossover at scale $L$ (or equivalently, the integral has an infrared cutoff at scale $L$ ), then the above expression will not be a pure power law of $r$, not even inside the inertial range. Then how is it possible that such an expression will cancel out a local term of $C(\boldsymbol{r})$, as is required by the typical equations of motion?

This puzzle has led in the past to the introduction of the concept of "window of locality" [9.12]. The window of locality is the range for the scaling exponents in which no divergence occurs, even if the crossover length $L$ is taken to infinity. For these exponents, integrals of type (1.1) are dominated by the range of integration $y \approx r$ and are therefore termed "local". In a "local" theory no infrared cutoff is called for.

In this paper we show that scaling behavior of the correlation functions together with finite integrals over an infinite domain do not necessarily imply a bounded spectrum of anisotropic exponents. Our strategy in this paper is to come up with a tractable example of a linear model with pressure, see Eq. (2.1). We refer to this model as the "linear pressure model". We approach the solutions of this model in two steps. First we distill yet another, simpler, exactly solvable model, which still poses the riddle of the Navier-Stokes problem. The exact solution reveals that the spectrum of scaling exponents is unbounded, and the convergence of the integrals is nevertheless not compromised. In the second step we find the scaling exponents of the "zero modes" 10,11] of the linear pressure model, and use the conclusions of the simpler model to relate them to the full solution. We show that also in this case the spectrum does not saturate in the anisotropic sectors.

The linear pressure model and its simplified version reveal two mechanisms that allow an unbounded spectrum of scaling exponents. First, a careful analysis of the window of locality in the anisotropic sectors shows that it widens as $\ell$ increases. We always have a leading scaling exponent within the window of locality. Second, there is a more subtle mechanism that comes to play when subleading exponents exist outside the window of locality. In these cases we show that there exist counter-terms in the exact solution (not the zero modes!) that maintain the locality of the integrals. The bottom line is that in these models the anisotropic exponents are unbounded from above leading to a fast decay of the anisotropic contributions in the inertial range.

In Sec. II we introduce the linear pressure model and derive the equations for the two-point correlation function. We arrive at the form containing the dangerous integrals, and discuss again the fundamental riddle. In Sec. III we construct a simpler, exactly solvable model with the same riddle. In Sec. IV we display the exact solution of the model, and discuss the windows of locality and the existence of an unbounded spectrum. In Sec. V we go back to the Linear Pressure model and offer a solution of its zero modes. Section V1 offers a summary and a discussion.

\section{LINEAR PRESSURE MODEL}

\section{A. The model}

The linear pressure model captures some of the aspects of the pressure term in Navier-Stokes turbulence, while being a linear and therefore, much simpler problem. The nonlinearity of the Navier-Stokes equation is replaced by an advecting field $\boldsymbol{w}(\boldsymbol{x}, t)$ and an advected field $\boldsymbol{v}(\boldsymbol{x}, t)$. The advecting field $\boldsymbol{w}(\boldsymbol{x}, t)$ is taken with known dynamics and statistics. Both the fields are assumed incompressible. The equation of motion for the vector field $v^{\alpha}(\boldsymbol{x}, t)$ is:

$$
\begin{aligned}
\partial_{t} v^{\alpha}+w^{\mu} \partial_{\mu} v^{\alpha}+\partial^{\alpha} p-\kappa \partial^{2} v^{\alpha} & =f^{\alpha}, \\
\partial_{\alpha} v^{\alpha} & =0, \\
\partial_{\alpha} w^{\alpha} & =0 .
\end{aligned}
$$

In this equation, $\boldsymbol{f}(\boldsymbol{x}, t)$ is a divergence free forcing term and $\kappa$ is the viscosity. The domain of the system is taken to be infinite. Following Kraichnan's model for passive scalar [0], we choose the advecting field $\boldsymbol{w}(\boldsymbol{x}, t)$ to be a white-noise Gaussian process with a correlation function that is given by

$$
\begin{aligned}
\delta\left(t^{\prime}-t\right) D^{\alpha \beta}(\boldsymbol{r}) & \equiv\left\langle w^{\alpha}\left(\boldsymbol{x}+\boldsymbol{r}, t^{\prime}\right) w^{\beta}(\boldsymbol{x}, t)\right\rangle, \\
K^{\alpha \beta}(\boldsymbol{r}) & \equiv D^{\alpha \beta}(\boldsymbol{r})-D^{\alpha \beta}(\mathbf{0}) \\
& =D r^{\xi}\left[(\xi+2) \delta^{\alpha \beta}-\xi \frac{r^{\alpha} r^{\beta}}{r^{2}}\right] .
\end{aligned}
$$

The forcing $\boldsymbol{f}(\boldsymbol{x}, t)$ is also taken to be a Gaussian white noise process. Its correlation function is

$$
F^{\alpha \beta}(\boldsymbol{r} / L) \delta\left(t-t^{\prime}\right) \equiv\left\langle f^{\alpha}(\boldsymbol{x}+\boldsymbol{r}, t) f^{\beta}\left(\boldsymbol{x}, t^{\prime}\right)\right\rangle .
$$

The forcing is responsible for injecting energy and anisotropy to the system at an outer scale $L$. We choose the tensor function $F^{\alpha \beta}(\boldsymbol{x})$ to be analytic in $\boldsymbol{x}$, anisotropic, and vanishing rapidly for $|\boldsymbol{x}| \gg 1$. Analyticity is an important requirement. It means that $F^{\alpha \beta}(\boldsymbol{x})$ can be expanded for small $|\boldsymbol{x}|$ as a power series in $x^{\alpha}$; as a result its leading contribution in the $\ell$ sector is proportional to $x^{\ell-2}$, given by $\partial^{\alpha} \partial^{\beta} x^{\ell} Y_{\ell m}(\hat{\boldsymbol{x}})$. To see that this is the leading contribution the reader can consult the general discussion of the construction of the irreducible representations in Ref. [1]. All other analytic contributions contain less derivatives and are therefore of higher order in $x$.

'In order to derive the statistical equations of the correlation function of $v^{\alpha}(\boldsymbol{x}, t)$, we need a version of Eq. (2.1) 
without the pressure term. Following the standard treatment of the pressure term in Navier-Stokes equation, we take the divergence of Eq. (2.1) and arrive at

$$
\partial_{\nu} \partial_{\mu} w^{\mu} v^{\nu}+\partial^{2} p=0 .
$$

The Laplace equation is now inverted using the Green function of infinite domain with zero-at-infinity boundary conditions,

$$
\begin{aligned}
p(\boldsymbol{x}) & =-\int d \boldsymbol{y} G(\boldsymbol{x}-\boldsymbol{y}) \partial_{\nu} \partial_{\mu} w^{\mu}(\boldsymbol{y}) v^{\nu}(\boldsymbol{y}), \\
G(\boldsymbol{x}) & \equiv-\frac{1}{4 \pi|\boldsymbol{x}|}
\end{aligned}
$$

With this expression for $p(\boldsymbol{x})$, Eq. 2.1) can be rewritten as

$$
\begin{aligned}
& \partial_{t} v^{\alpha}(\boldsymbol{x}, t)+w^{\mu}(\boldsymbol{x}, t) \partial_{\mu} v^{\alpha}(\boldsymbol{x}, t) \\
& \quad-\partial_{(\boldsymbol{x})}^{\alpha} \int d \boldsymbol{y} G(\boldsymbol{x}-\boldsymbol{y}) \partial_{\nu} \partial_{\mu} w^{\mu}(\boldsymbol{y}) v^{\nu}(\boldsymbol{y}) \\
& -\kappa \partial^{2} v^{\alpha}(\boldsymbol{x}, t)=f^{\alpha}(\boldsymbol{x}, t)
\end{aligned}
$$

\section{B. Equations for the second-order correlation function}

The statistical object that we are interested in is the two-point correlation function of the field $v^{\alpha}(\boldsymbol{x})$,

$$
C^{\alpha \beta}(\boldsymbol{r}) \equiv\left\langle v^{\alpha}(\boldsymbol{x}+\boldsymbol{r}) v^{\beta}(\boldsymbol{x})\right\rangle .
$$

We find its equation of motion in two steps. First, we take the time derivative of $C^{\alpha \beta}(\boldsymbol{r})$ using Eq. (2.11),

$$
\begin{aligned}
& \partial_{t}\left\langle v^{\alpha}(\boldsymbol{x}+\boldsymbol{r}) v^{\beta}(\boldsymbol{x})\right\rangle+\left\langle v^{\alpha}(\boldsymbol{x}+\boldsymbol{r}) w^{\mu}(\boldsymbol{x}) \partial_{\mu} v^{\beta}(\boldsymbol{x})\right\rangle \\
& +\left\langle v^{\beta}(\boldsymbol{x}) w^{\mu}(\boldsymbol{x}+\boldsymbol{r}) \partial_{\mu} v^{\alpha}(\boldsymbol{x}+\boldsymbol{r})\right\rangle \\
& -\left\langle v^{\alpha}(\boldsymbol{x}+\boldsymbol{r}) \partial_{(x)}^{\beta} \int d \boldsymbol{y} G(\boldsymbol{x}-\boldsymbol{y}) \partial_{\mu} \partial_{\nu} w^{\mu}(\boldsymbol{y}) v^{\nu}(\boldsymbol{y})\right\rangle \\
& -\left\langle v^{\beta}(\boldsymbol{x}) \partial_{(x+r)}^{\alpha} \int d \boldsymbol{y} G(\boldsymbol{x}+\boldsymbol{r}-\boldsymbol{y}) \partial_{\mu} \partial_{\nu} w^{\mu}(\boldsymbol{y}) v^{\nu}(\boldsymbol{y})\right\rangle \\
& -\kappa\left\langle v^{\alpha}(\boldsymbol{x}+\boldsymbol{r}) \partial^{2} v^{\beta}(\boldsymbol{x})\right\rangle-\kappa\left\langle v^{\beta}(\boldsymbol{x}) \partial^{2} v^{\alpha}(\boldsymbol{x}+\boldsymbol{r})\right\rangle \\
& =\left\langle v^{\alpha}(\boldsymbol{x}+\boldsymbol{r}) f^{\beta}(\boldsymbol{x})\right\rangle+\left\langle v^{\beta}(\boldsymbol{x}) f^{\alpha}(\boldsymbol{x}+\boldsymbol{r})\right\rangle .
\end{aligned}
$$

To simplify the equations we define an auxiliary function $T^{\alpha \beta}(\boldsymbol{r})$

$$
T^{\alpha \beta}(\boldsymbol{r}) \equiv \partial_{\mu}^{(r)}\left\langle v^{\alpha}(\boldsymbol{x}+\boldsymbol{r}) w^{\mu}(\boldsymbol{x}) v^{\beta}(\boldsymbol{x})\right\rangle .
$$

Using this definition and the space homogeneity of the statistics, we arrive after some algebraic manipulation to the following equation:

$$
\begin{aligned}
& \partial_{t} C^{\alpha \beta}(\boldsymbol{r})-T^{\alpha \beta}(\boldsymbol{r})-T^{\beta \alpha}(-\boldsymbol{r}) \\
& +\int d \boldsymbol{y} G(\boldsymbol{r}-\boldsymbol{y}) \partial^{\beta} \partial_{\nu} T^{\alpha \nu}(\boldsymbol{y})+\int d \boldsymbol{y} G(-\boldsymbol{r}-\boldsymbol{y}) \partial^{\alpha} \partial_{\nu} T^{\beta \nu}(\boldsymbol{y}) \\
& -2 \kappa \partial^{2} C^{\alpha \beta}(\boldsymbol{r})=\left\langle v^{\alpha}(\boldsymbol{x}+\boldsymbol{r}) f^{\beta}(\boldsymbol{x})\right\rangle+\left\langle v^{\beta}(\boldsymbol{x}) f^{\alpha}(\boldsymbol{x}+\boldsymbol{r})\right\rangle .
\end{aligned}
$$

The last equation is identical to the equation for the second-order correlation function in the usual NavierStokes turbulence, provided that $w^{\mu}$ is replaced with $v^{\mu}$ in Eq. (2.16). Indeed, the vexing problem that we face is being made very clear: if the triple correlation function has a power law dependence on $\boldsymbol{r}$ with an arbitrarily large exponent, how can the integral converge in the infrared? One possibility is that the scaling exponent of $T^{\alpha \beta}(\boldsymbol{r})$ is sufficiently low, making the integral convergent. The other possibility is that the correlation function is scale invariant only in the inertial range and vanishes quickly after that, which is equivalent to the introduction of an infrared cutoff. However, the integral terms in the equation probe the correlation function throughout the entire space. Therefore, a crossover behavior of the correlation function at the outer scale $L$, seems to contradict a pure scaling behavior of the correlation function in the inertial range itself. This in turn implies the saturation of the anisotropic scaling exponents.

To proceed, we use the fact that the field $\boldsymbol{w}(\boldsymbol{x}, t)$, as well as the forcing, are Gaussian white noises. This enables us to express $T^{\alpha \beta}(\boldsymbol{r})$ and the correlation of the force in terms of $C^{\alpha \beta}(\boldsymbol{r})$ and $F^{\alpha \beta}(\boldsymbol{r})$. In appendix A we use the well-known method of Gaussian integration by parts [13] that leads to the final equations,

$$
\begin{aligned}
\partial_{t} C^{\alpha \beta}(\boldsymbol{r})= & T^{\alpha \beta}(\boldsymbol{r})+T^{\beta \alpha}(-\boldsymbol{r}) \\
& -\int d \boldsymbol{y} G(\boldsymbol{r}-\boldsymbol{y}) \partial^{\beta} \partial_{\nu} T^{\alpha \nu}(\boldsymbol{y}) \\
& -\int d \boldsymbol{y} G(-\boldsymbol{r}-\boldsymbol{y}) \partial^{\alpha} \partial_{\nu} T^{\beta \nu}(\boldsymbol{y}) \\
& +2 \kappa \partial^{2} C^{\alpha \beta}(\boldsymbol{r})+F^{\alpha \beta}(\boldsymbol{r}) \\
T^{\alpha \beta}(\boldsymbol{r})= & -\frac{1}{2} K^{\mu \nu} \partial_{\mu} \partial_{\nu} C^{\alpha \beta}(\boldsymbol{r}) \\
& +\frac{1}{2} \partial_{(\boldsymbol{r})}^{\alpha} \int d \boldsymbol{y} G(\boldsymbol{r}-\boldsymbol{y}) \partial_{\tau}\left[K^{\mu \nu}(\boldsymbol{y}) \partial_{\mu} \partial_{\nu} C^{\tau \beta}(\boldsymbol{y})\right] \\
& -\frac{1}{2} \int d \boldsymbol{y} G(\boldsymbol{y}) \partial^{\beta} \partial_{\tau}\left[K^{\mu \nu}(\boldsymbol{y}) \partial_{\mu} \partial_{\nu} C^{\alpha \tau}(\boldsymbol{r}-\boldsymbol{y})\right]
\end{aligned}
$$

These equations have to be supplemented with two more equations that follow directly from the definition of $C^{\alpha \beta}(\boldsymbol{r})$,

$$
\begin{aligned}
\partial_{\alpha} C^{\alpha \beta}(\boldsymbol{r}) & =0, \\
C^{\alpha \beta}(\boldsymbol{r}) & =C^{\beta \alpha}(-\boldsymbol{r}) .
\end{aligned}
$$

The first equation follows from the incompressibility constraint of the vector field $\boldsymbol{v}(\boldsymbol{x}, t)$, while the latter follows from space homogeneity. Finally, we note that Eqs. (2.18) and (2.19) can be interpreted in a transparent way, utilizing two projection operators that maintain the right-hand side (RHS) of Eq. (2.18) divergence-free in both indices. To define them, let us consider a tensor field $X^{\alpha \beta}(\boldsymbol{r})$ that vanishes sufficiently fast at infinity. 
Then the two projection operators $\hat{\mathcal{P}}_{\mathrm{L}}$ and $\hat{\mathcal{P}}_{\mathrm{R}}$ are defined by

$$
\begin{aligned}
& \hat{\mathcal{P}}_{\mathrm{L}} X^{\alpha \beta}(\boldsymbol{r}) \equiv X^{\alpha \beta}(\boldsymbol{r})-\partial_{(r)}^{\alpha} \int d \boldsymbol{y} G(\boldsymbol{r}-\boldsymbol{y}) \partial_{\mu} X^{\mu \beta}(\boldsymbol{y}) \\
& \hat{\mathcal{P}}_{\mathrm{R}} X^{\alpha \beta}(\boldsymbol{r}) \equiv X^{\alpha \beta}(\boldsymbol{r})-\partial_{(r)}^{\beta} \int d \boldsymbol{y} G(\boldsymbol{r}-\boldsymbol{y}) \partial_{\mu} X^{\alpha \mu}(\boldsymbol{y})
\end{aligned}
$$

We observe that $\hat{\mathcal{P}}_{\mathrm{L}} X^{\alpha \beta}$ and $\hat{\mathcal{P}}_{\mathrm{R}} X^{\alpha \beta}$ are divergence-free in the left and right indices, respectively. Using these operators we can rewrite Eqs.(2.182.19) in the form

$$
\begin{aligned}
\partial_{t} C^{\alpha \beta}(\boldsymbol{r}) & =\hat{\mathcal{P}}_{\mathrm{R}} T^{\alpha \beta}(\boldsymbol{r})+\hat{\mathcal{P}}_{\mathrm{R}} T^{\beta \alpha}(-\boldsymbol{r}) \\
& +2 \kappa \partial^{2} C^{\alpha \beta}(\boldsymbol{r})+F^{\alpha \beta}(\boldsymbol{r}), \\
T^{\alpha \beta}(\boldsymbol{r}) & =-\frac{1}{2} \hat{\mathcal{P}}_{\mathrm{L}} K^{\mu \nu} \partial_{\mu} \partial_{\nu} C^{\alpha \beta}(\boldsymbol{r})- \\
& -\frac{1}{2} \int d \boldsymbol{y} G(\boldsymbol{y}) \partial^{\beta} \partial_{\tau}\left[K^{\mu \nu}(\boldsymbol{y}) \partial_{\mu} \partial_{\nu} C^{\alpha \tau}(\boldsymbol{r}-\boldsymbol{y})\right] .
\end{aligned}
$$

The projection in Eq. (2.25) guarantees that $T^{\alpha \beta}(\boldsymbol{r})$ is divergence free in its left index, while the projection in Eq. (2.24) guarantees divergence freedom in the right index.

Not all the terms in these equations are of the same nature. The integrals due to the projection operator are easy to deal with by applying a Laplacian on them. For example,

$$
\partial^{2} \hat{\mathcal{P}}_{\mathrm{R}} T^{\alpha \beta}(\boldsymbol{r})=\partial^{2} T^{\alpha \beta}(\boldsymbol{r})-\partial^{\beta} \partial_{\nu} T^{\alpha \nu}(\boldsymbol{r}) .
$$

On the other hand, there seems to be no way to eliminate the last integral in Eq. (2.25), and therefore we shall refer to it as the "nontrivial integral". Only for $\xi=0$ and $\xi=2$ it trivializes: the integral vanishes when $\xi=0$ and is proportional to $C^{\alpha \beta}(\boldsymbol{r})$ when $\xi=2$. Unfortunately, in these extreme cases also the projection operator trivializes, and the effect of the pressure cannot be adequately assessed. We prefer to study the problem for a generic value $\xi$ for which the incompressibility constraint and the pressure terms are nontrivial.

We deal with the this problem head-on in Sec. 凤. Due to the nontrivial integral, we will not be able to provide a full solution of $C^{\alpha \beta}(\boldsymbol{r})$, but only of the zero modes. However, before doing so we would like to study a model that affords an exact solution in order to understand in detail the issues at hand. In the next section we therefore consider a simplified model of the linear pressure model, yet posing much of the same riddle.

\section{AN EXACTLY SOLVABLE TOY MODEL}

We construct a toy model that inspired by Eqs. 2.18 and, (2.19) for the correlation function in the linear pressure model. Within this model we demonstrate the strategy of dealing with the nonlocal-pressure term. Since it is a simplification of the statistical equation of the linear pressure model, the toy model has no obvious underlying dynamical equation.

\section{A. Definition of the toy model}

In the toy model, we are looking for a "correlation function" $C^{\alpha}(\boldsymbol{r})$, whose equations of motion are

$$
\begin{aligned}
\partial_{t} C^{\alpha}(\boldsymbol{r})= & -K^{\mu \nu}(\boldsymbol{r}) \partial_{\mu} \partial_{\nu} C^{\alpha}(\boldsymbol{r})-\partial_{(r)}^{\alpha} \\
& \times \int d \boldsymbol{x} G(\boldsymbol{r}-\boldsymbol{x}) \partial_{\tau} K^{\mu \nu}(\boldsymbol{x}) \partial_{\mu} \partial_{\nu} C^{\tau}(\boldsymbol{x}) \\
& +\kappa \partial^{2} C^{\alpha}(\boldsymbol{r})+F^{\alpha}(\boldsymbol{r} / L), \\
\partial_{\alpha} C^{\alpha}(\boldsymbol{r})= & 0 .
\end{aligned}
$$

Here $F^{\alpha}(\boldsymbol{x})$ is a one-index analog of the correlation function of the original forces $F^{\alpha \beta}(\boldsymbol{x})$. Accordingly, we take it anisotropic, analytic in $x^{\alpha}$ and rapidly vanishing for $|\boldsymbol{x}| \gg 1$. As in the previous model, also here analyticity requires that the leading contribution for small $|\boldsymbol{x}|$ is proportional to $\partial^{\alpha} x^{\ell} Y_{\ell m}(\hat{\boldsymbol{x}})$ in the $\ell$ sector. Accordingly it is of order $x^{\ell-1}$.

The toy model is simpler than the Linear Pressure model in two aspects: First, the "correlation function", $C^{\alpha}(\boldsymbol{r})$ has one index instead of two and therefore can be represented by a smaller number of scalar functions. Second, the unpleasant nontrivial term of the linear pressure model is absent. This will allow us to solve the model exactly for every value of $\xi$. Nevertheless, the toy model confronts us with the same conceptual problems that exist in the linear pressure model and in NS turbulence: can a scale invariant solution in the inertial range with a crossover to a decaying solution at scale $L$, be consistent with the integral term? If not, is there a saturation of the anisotropic exponents?

Equation 3.1 can be rewritten in terms of a new projection operator $\hat{\mathcal{P}}$, which projects a vector $X^{\alpha}(\boldsymbol{r})$ on its divergence free part

$$
\partial_{t} C^{\alpha}=-\hat{\mathcal{P}}\left[K^{\mu \nu} \partial_{\mu} \partial_{\nu} C^{\alpha}\right]+\kappa \partial^{2} C^{\alpha}+F^{\alpha}
$$

where

$$
\hat{\mathcal{P}} X^{\alpha}(\boldsymbol{r}) \equiv X^{\alpha}(\boldsymbol{r})-\partial^{\alpha} \int d \boldsymbol{y} G(\boldsymbol{r}-\boldsymbol{y}) \partial_{\mu} X^{\mu}(\boldsymbol{y})
$$

We shall solve this integro-differential equation by first turning it into a partial differentail equation (PDE) using the Laplacian operator, and then turning it into a set of decoupled ODE's using the $\mathrm{SO}(3)$ decomposition.

As in the linear pressure model, the nonlocality of the projection operator can be removed by considering a differential version of it

$$
\partial^{2} \hat{\mathcal{P}} T^{\alpha}(\boldsymbol{r})=\partial^{2} T^{\alpha}(\boldsymbol{r})-\partial^{\alpha} \partial_{\mu} T^{\mu}(\boldsymbol{r})
$$

In stationary condition $\partial_{t} C^{\alpha}=0$, and therefore the differential form of the toy model is given by 


$$
\begin{aligned}
& \partial^{2} \hat{\mathcal{P}}\left[K^{\mu \nu}(\boldsymbol{r}) \partial_{\mu} \partial_{\nu} C^{\alpha}(\boldsymbol{r})\right] \\
& \quad=\partial^{2} K^{\mu \nu}(\boldsymbol{r}) \partial_{\mu} \partial_{\nu} C^{\alpha}(\boldsymbol{r})-\partial^{\alpha} \partial_{\tau} K^{\mu \nu}(\boldsymbol{r}) \partial_{\mu} \partial_{\nu} C^{\tau}(\boldsymbol{r}) \\
& \quad=\kappa \partial^{2} \partial^{2} C^{\alpha}+\partial^{2} F^{\alpha} \\
& \partial_{\alpha} C^{\alpha}(\boldsymbol{r})=0 .
\end{aligned}
$$

We have reached a linear PDE of order four. This PDE will be solved by exploiting its symmetries, i.e., isotropy and parity conservation, as demonstrated in the next subsection.

\section{B. The $\mathbf{S O}(3)$ decomposition}

Equation 3.6 and the incompressibility condition of $C^{\alpha}(\boldsymbol{r})$ are both isotropic and parity conserving. Therefore, if we expand $C^{\alpha}(\boldsymbol{r})$ in terms of spherical vectors with a definite behavior under rotations and under reflections, we would get a set of decoupled ODE's for their coefficients. For each sector $(\ell, m), \ell>0$ of $\mathrm{SO}(3)$ we have three spherical vectors:

$$
\begin{aligned}
A_{1}^{\alpha}(\hat{\boldsymbol{r}}) & \equiv r^{-\ell-1} r^{\alpha} \Phi_{\ell m}(\boldsymbol{r}) \\
A_{2}^{\alpha}(\hat{\boldsymbol{r}}) & \equiv r^{-\ell+1} \partial^{\alpha} \Phi_{\ell m}(\boldsymbol{r}) \\
A_{3}^{\alpha}(\hat{\boldsymbol{r}}) & \equiv r^{-\ell} \epsilon^{\alpha \mu \nu} r_{\mu} \partial_{\nu} \Phi_{\ell m}(\boldsymbol{r}) .
\end{aligned}
$$

Here $\Phi_{\ell m}(\boldsymbol{r})=r^{\ell} Y_{\ell m}(\hat{\boldsymbol{r}})$, and see [1] for further details. The first two spherical vectors have a different parity than the third vector, hence the equations for their coefficients are decoupled from the equation for the third coefficient. In the following, we shall consider the equations for the first two coefficients only, as they have a richer structure and larger resemblance to the linear pressure model. Finally, note that the isotropic sector, i.e., $\ell=0$, is identically zero. To see why, notice that in this special sector there is only one spherical vector, $A_{1}^{\alpha}(\hat{\boldsymbol{r}}) \equiv r^{-1} r^{\alpha}$. Hence the isotropic part of $C^{\alpha}(\boldsymbol{r})$ is given by $c(r) r^{-1} r^{\alpha}$, $c(r)$ being some scalar function of $r$. But then the incompressibility condition (3.2) implies that $c(r) \sim r^{-2}$, which has a UV divergence. We therefore conclude that $c(r)=0$, and restrict our calculation to $\ell>0$.

By expanding $C^{\alpha}(\boldsymbol{r})$ in terms of the spherical vectors $\boldsymbol{A}_{1}$ and $\boldsymbol{A}_{2}$, we obtain a set of ODEs [decoupled in the $(\ell, m)$ labels] for the scalar functions that are the coefficients of these vectors in the expansion. The equations for these coefficients can thus be written in terms of matrices and column vectors. To simplify the calculations, we find the matrix forms of the Kraichnan operator and of the Laplacian of the projection operator separately, and only then combine the two results to one.

\section{The matrix form of the Kraichnan operator}

To obtain the matrix of the Kraichnan operator in the basis of $\boldsymbol{A}_{1}$ and $\boldsymbol{A}_{2}$, we expand $C^{\alpha}(\boldsymbol{r})$ :

$$
C^{\alpha}(\boldsymbol{r})=c_{1}(r) A_{1}^{\alpha}(\hat{\boldsymbol{r}})+c_{2}(r) A_{2}^{\alpha}(\hat{\boldsymbol{r}}) .
$$

Using the basic identities of the $\Phi_{\ell m}(\boldsymbol{r})$ functions (see [1]),

$$
\begin{aligned}
\partial^{2} \Phi_{\ell m}(\boldsymbol{r}) & =0, \\
r^{\mu} \partial_{\mu} \Phi_{\ell m}(\boldsymbol{r}) & =\ell \Phi_{\ell m}(\boldsymbol{r}),
\end{aligned}
$$

a short calculation yields

$$
\begin{aligned}
\hat{\mathcal{K}} & C^{\alpha}(\boldsymbol{r}) \equiv K^{\mu \nu}(\boldsymbol{r}) \partial_{\mu} \partial_{\nu} C^{\alpha}(\boldsymbol{r}) \\
= & D x^{\xi}\left[2 c_{1}^{\prime \prime}+2(2+\xi) \frac{c_{1}^{\prime}}{r}-(2+\xi)(\ell+1)(\ell+2) \frac{c_{1}}{r^{2}}\right] A_{1}^{\alpha}(\hat{\boldsymbol{r}}) \\
& +D x^{\xi}\left[2 c_{2}^{\prime \prime}+2(2+\xi) \frac{c_{2}^{\prime}}{r}+2(2+\xi) \frac{c_{1}}{r^{2}}\right. \\
& \left.-2(2+\xi) \ell(\ell-1) \frac{c_{2}}{r^{2}}\right] A_{2}^{\alpha}(\hat{\boldsymbol{r}}) .
\end{aligned}
$$

Therefore, in matrix notation, the Kraichnan operator can be written as:

$$
\begin{aligned}
\hat{\mathcal{K}} & \left(\begin{array}{l}
c_{1} \\
c_{2}
\end{array}\right)=2 D r^{\xi}\left(\begin{array}{ll}
1 & 0 \\
0 & 1
\end{array}\right)\left(\begin{array}{l}
c_{1}^{\prime \prime} \\
c_{2}^{\prime \prime}
\end{array}\right) \\
& +2 D(2+\xi) r^{\xi-1}\left(\begin{array}{ll}
1 & 0 \\
0 & 1
\end{array}\right)\left(\begin{array}{l}
c_{1}^{\prime} \\
c_{2}^{\prime}
\end{array}\right) \\
& -D(2+\xi) r^{\xi-2}\left(\begin{array}{cc}
(\ell+1)(\ell+2) & 0 \\
-2 & \ell(\ell-1)
\end{array}\right)\left(\begin{array}{l}
c_{1} \\
c_{2}
\end{array}\right) \\
\equiv & r^{\xi} \mathbb{K}_{2}\left(\begin{array}{c}
c_{1}^{\prime \prime} \\
c_{2}^{\prime \prime}
\end{array}\right)+r^{\xi-1} \mathbb{K}_{1}\left(\begin{array}{c}
c_{1}^{\prime} \\
c_{2}^{\prime}
\end{array}\right)+r^{\xi-2} \mathbb{K}_{0}\left(\begin{array}{l}
c_{1} \\
c_{2}
\end{array}\right) .
\end{aligned}
$$

\section{The matrix form of the Laplacian of the Projection} operator

Let

$$
T^{\alpha}(\boldsymbol{r})=t_{1}(r) A_{1}^{\alpha}(\hat{\boldsymbol{r}})+t_{2}(r) A_{2}^{\alpha}(\hat{\boldsymbol{r}})
$$

and applying a Laplacian to $\hat{\mathcal{P}} T^{\alpha}$, we get

$$
\begin{aligned}
& \partial^{2} \hat{\mathcal{P}} T^{\alpha}=\left[-\ell t_{2}^{\prime \prime}+\ell \frac{t_{1}^{\prime}}{r}+\ell(2 \ell-1) \frac{t_{2}^{\prime}}{r}\right. \\
& \left.\quad-\ell(\ell+1) \frac{t_{1}}{r^{2}}-\ell(\ell-1)(\ell+1) \frac{t_{2}}{r^{2}}\right] A_{1}^{\alpha} \\
& +\left[t_{2}^{\prime \prime}-\frac{t_{1}^{\prime}}{r}+(2-\ell) \frac{t_{2}^{\prime}}{r}\right] A_{2}^{\alpha} .
\end{aligned}
$$

Hence in matrix notation,

$$
\begin{aligned}
\partial^{2} \hat{\mathcal{P}}\left(\begin{array}{l}
t_{1} \\
t_{2}
\end{array}\right)=\left(\begin{array}{cc}
0 & -\ell \\
0 & 1
\end{array}\right)\left(\begin{array}{c}
t_{1}^{\prime \prime} \\
t_{2}^{\prime \prime}
\end{array}\right) \\
+\frac{1}{r}\left(\begin{array}{cc}
\ell & \ell(2 \ell-1) \\
-1 & 2-\ell
\end{array}\right)\left(\begin{array}{c}
t_{1}^{\prime} \\
t_{2}^{\prime}
\end{array}\right) \\
-\frac{1}{r^{2}}\left(\begin{array}{cc}
\ell(\ell+1) & \ell(\ell-1)(\ell+1) \\
0 & 0
\end{array}\right)\left(\begin{array}{c}
t_{1} \\
t_{2}
\end{array}\right) \\
\equiv \mathbb{P}_{2}\left(\begin{array}{c}
t_{1}^{\prime \prime} \\
t_{2}^{\prime \prime}
\end{array}\right)+\frac{1}{r} \mathbb{P}_{1}\left(\begin{array}{c}
t_{1}^{\prime} \\
t_{2}^{\prime}
\end{array}\right)+\frac{1}{r^{2}} \mathbb{P}_{0}\left(\begin{array}{c}
t_{1} \\
t_{2}
\end{array}\right) .
\end{aligned}
$$




\section{The matrix form of the toy model}

Now that the matrix forms of the Kraichnan operator and of the Laplacian of the projection operator have been found, we can combine these two results to find the matrix form of the left-hand side (LHS) of Eq. (3.6). To this aim let us define

$$
\left(\begin{array}{l}
t_{1} \\
t_{2}
\end{array}\right)=\hat{\mathcal{K}}\left(\begin{array}{l}
c_{1} \\
c_{2}
\end{array}\right)
$$

and from Eqs. (3.11) and (3.14) we get

$$
\begin{aligned}
& \partial^{2} \hat{\mathcal{P}} \hat{\mathcal{K}}\left(\begin{array}{c}
c_{1} \\
c_{2}
\end{array}\right)=r^{\xi} \mathbb{M}_{4}\left(\begin{array}{c}
c_{1}^{(4)} \\
c_{2}^{(4)}
\end{array}\right)+r^{\xi-1} \mathbb{M}_{3}\left(\begin{array}{c}
c_{1}^{(3)} \\
c_{2}^{(3)}
\end{array}\right) \\
& +r^{\xi-2} \mathbb{M}_{2}\left(\begin{array}{c}
c_{1}^{(2)} \\
c_{2}^{(2)}
\end{array}\right)+r^{\xi-3} \mathbb{M}_{1}\left(\begin{array}{c}
c_{1}^{(1)} \\
c_{2}^{(1)}
\end{array}\right) \\
& +r^{\xi-4} \mathbb{M}_{0}\left(\begin{array}{c}
c_{1} \\
c_{2}
\end{array}\right),
\end{aligned}
$$

where the number in parentheses denotes the order of the derivative. The matrices $\mathbb{M}_{i}$ are given by

$$
\begin{aligned}
\mathbb{M}_{4} \equiv & \mathbb{P}_{2} \mathbb{K}_{2} \\
\mathbb{M}_{3} \equiv & 2 \xi \mathbb{P}_{2} \mathbb{K}_{2}+\mathbb{P}_{2} \mathbb{K}_{1}+\mathbb{P}_{1} \mathbb{K}_{2} \\
\mathbb{M}_{2} \equiv & \xi(\xi-1) \mathbb{P}_{2} \mathbb{K}_{2}+2(\xi-1) \mathbb{P}_{2} \mathbb{K}_{1}+\mathbb{P}_{2} \mathbb{K}_{0} \\
& +\xi \mathbb{P}_{1} \mathbb{K}_{2}+\mathbb{P}_{1} \mathbb{K}_{1}+\mathbb{P}_{0} \mathbb{K}_{2} \\
\mathbb{M}_{1} \equiv & (\xi-1)(\xi-2) \mathbb{P}_{2} \mathbb{K}_{1}+2(\xi-2) \mathbb{P}_{2} \mathbb{K}_{0} \\
& +(\xi-1) \mathbb{P}_{1} \mathbb{K}_{1}+\mathbb{P}_{1} \mathbb{K}_{0}+\mathbb{P}_{0} \mathbb{K}_{1} \\
\mathbb{M}_{0} \equiv & (\xi-2)(\xi-3) \mathbb{P}_{2} \mathbb{K}_{0}+(\xi-2) \mathbb{P}_{1} \mathbb{K}_{0}+\mathbb{P}_{0} \mathbb{K}_{0}
\end{aligned}
$$

To find the RHS of Eq. (3.6) we expand the "forcing" $F^{\alpha}(\boldsymbol{r})$ in terms of the spherical vectors $\boldsymbol{A}_{1}$ and $\boldsymbol{A}_{2}$,

$$
F^{\alpha}(\boldsymbol{r})=f_{1}(r) A_{1}^{\alpha}(\hat{\boldsymbol{r}})+f_{2}(r) A_{2}^{\alpha}(\hat{\boldsymbol{r}}),
$$

and applying a Laplacian we find the matrix form of $\partial^{2} F^{\alpha}(\boldsymbol{r})$,

$$
\begin{aligned}
& \partial^{2}\left(\begin{array}{l}
f_{1} \\
f_{2}
\end{array}\right)=\left(\begin{array}{c}
f_{1}^{\prime \prime}+\frac{2}{r} f_{1}^{\prime}-(\ell+1)(\ell+2) \frac{1}{r^{2}} f_{1} \\
f_{2}^{\prime \prime}+\frac{2}{r} f_{2}^{\prime}+\frac{2}{r^{2}} f_{1}-\ell(\ell-1) \frac{1}{r^{2}} f_{2}
\end{array}\right) \\
& \equiv\left(\begin{array}{l}
\rho_{1} \\
\rho_{2}
\end{array}\right) .
\end{aligned}
$$

At this point it is worthwhile to remember that the forcing term $F^{\alpha}(\boldsymbol{r} / L)$ is assumed to be analytic. As a result for $r / L \ll 1$, its leading contribution in the $(\ell, m)$ sector is proportional to $\partial^{\alpha} r^{\ell} Y_{\ell m}(\hat{\boldsymbol{r}}) \sim r^{\ell-1}$. However $\partial^{2} F^{\alpha}(\boldsymbol{r} / L)$ is also analytic, and must therefore also scale like $r^{\ell-1}$ for small $r$, instead of scaling like $r^{\ell-3}$, which could be the naive dimensional guess.

To proceed we restrict ourselves to finding the solution in the inertial range and beyond. In these ranges the dissipative term $\kappa \partial^{2} \partial^{2} C^{\alpha}(\boldsymbol{r})$ is negligible and can be omitted, thus reaching the following equation for the column vector $\left(c_{1}, c_{2}\right)$ :

$$
\begin{gathered}
r^{\xi} \mathbb{M}_{4}\left(\begin{array}{c}
c_{1}^{(4)} \\
c_{2}^{(4)}
\end{array}\right)+r^{\xi-1} \mathbb{M}_{3}\left(\begin{array}{c}
c_{1}^{(3)} \\
c_{2}^{(3)}
\end{array}\right)+r^{\xi-2} \mathbb{M}_{2}\left(\begin{array}{c}
c_{1}^{(2)} \\
c_{2}^{(2)}
\end{array}\right) \\
+r^{\xi-3} \mathbb{M}_{1}\left(\begin{array}{c}
c_{1}^{(1)} \\
c_{2}^{(1)}
\end{array}\right)+r^{\xi-4} \mathbb{M}_{0}\left(\begin{array}{c}
c_{1} \\
c_{2}
\end{array}\right)=\left(\begin{array}{c}
\rho_{1} \\
\rho_{2}
\end{array}\right) \cdot
\end{gathered}
$$

Finally, also the incompressibility constraint $\partial_{\alpha} C^{\alpha}(\boldsymbol{r})=0$, can be expressed as a relation between $c_{1}(r)$ and $c_{2}(r)$

$$
c_{1}^{\prime}+2 \frac{c_{1}}{r}+\ell c_{2}^{\prime}-\ell(\ell-1) \frac{c_{2}}{r}=0 .
$$

This constraint has to be taken into account when solving Eq. (3.20).

\section{SOLVING THE TOY MODEL}

\section{A. The general solution}

The solution of Eq. (3.20) is somewhat tricky due to the additional constraint (3.21). Seemingly the two unknowns $c_{1}(r)$ and $c_{2}(r)$ are over determined by the three equations (3.20, 3.21), yet this is not the case for the two equations (3.20) are not independent (when considered as two scalar equations, resulting from the two-column vectorial equation). To see that this is the case and find the solution, it is advantageous to work in the new basis

$$
\begin{aligned}
& d_{1}=c_{1}+\ell c_{2}, \\
& d_{2}=-2 c_{1}+\ell(\ell-1) c_{2} .
\end{aligned}
$$

In this basis the incompressibility constraint becomes very simple,

$$
d_{2}=r d_{1}^{\prime}
$$

allowing us to express $d_{2}$ and its derivatives in terms of $d_{1}$. To do that in the framework of the matrix notation, we define the transformation matrix $\mathbb{U}$

$$
\begin{aligned}
\mathbb{U} & \equiv\left(\begin{array}{cc}
1 & \ell \\
-2 & \ell(\ell-1)
\end{array}\right), \\
\mathbb{U}^{-1} & =\frac{1}{\ell(\ell+1)}\left(\begin{array}{cc}
\ell(\ell-1) & -\ell \\
2 & 1
\end{array}\right),
\end{aligned}
$$

so that,

$$
\left(\begin{array}{l}
d_{1} \\
d_{2}
\end{array}\right)=\mathbb{U}\left(\begin{array}{l}
c_{1} \\
c_{2}
\end{array}\right) .
$$

The equations of $d_{i}(r)$ are the same as the equations for $c_{i}(r)$, with the matrices $\mathbb{M}_{i}$ replaced by

$$
\mathbb{N}_{i} \equiv \mathbb{U M}_{i} \mathbb{U}^{-1}
$$


and the sources $\rho_{i}$ replaced by

$$
\left(\begin{array}{c}
\rho_{1}^{*} \\
\rho_{2}^{*}
\end{array}\right)=\mathbb{U}\left(\begin{array}{l}
\rho_{1} \\
\rho_{2}
\end{array}\right)
$$

Notice that a divergence free forcing $F^{\alpha}(\boldsymbol{r})$ will cause $\rho_{1}^{*}(r)$ and $\rho_{2}^{*}(r)$ to be related to each other in the same way that $d_{1}(r)$ and $d_{2}(r)$ are related to each other, i.e.,

$$
\rho_{2}^{*}=r\left(\rho_{1}^{*}\right)^{\prime} .
$$

Next, we perform the following replacements:

$$
\begin{aligned}
d_{2} & =r d_{1}^{(1)}, \\
d_{2}^{(1)} & =r d_{1}^{(2)}+d_{1}^{(1)}, \\
d_{2}^{(2)} & =r d_{1}^{(3)}+2 d_{1}^{(2)}, \\
d_{2}^{(3)} & =r d_{1}^{(4)}+3 d_{1}^{(3)}, \\
d_{2}^{(4)} & =r d_{1}^{(5)}+4 d_{1}^{(3)} .
\end{aligned}
$$

We get an equation written entirely in terms of the function $d_{1}(r)$ and its derivatives,

$$
\begin{aligned}
& r^{\xi+1} V_{5} d_{1}^{(5)}+r^{\xi} V_{4} d_{1}^{(4)}+r^{\xi-1} V_{3} d_{1}^{(3)}+r^{\xi-2} V_{2} d_{1}^{(2)} \\
& +r^{\xi-3} V_{1} d_{1}^{(1)}+r^{\xi-4} V_{0} d_{1}=\left(\begin{array}{c}
\rho_{1}^{*} \\
\rho_{2}^{*}
\end{array}\right)
\end{aligned}
$$

where $V_{i}$ are two dimensional vectors given by,

$$
\begin{aligned}
& V_{5} \equiv \mathbb{N}_{4}\left(\begin{array}{l}
0 \\
1
\end{array}\right), \\
& V_{4} \equiv \mathbb{N}_{4}\left(\begin{array}{l}
1 \\
4
\end{array}\right)+\mathbb{N}_{3}\left(\begin{array}{l}
0 \\
1
\end{array}\right), \\
& V_{3} \equiv \mathbb{N}_{3}\left(\begin{array}{l}
1 \\
3
\end{array}\right)+\mathbb{N}_{2}\left(\begin{array}{l}
0 \\
1
\end{array}\right), \\
& V_{2} \equiv \mathbb{N}_{2}\left(\begin{array}{l}
1 \\
2
\end{array}\right)+\mathbb{N}_{1}\left(\begin{array}{l}
0 \\
1
\end{array}\right), \\
& V_{1} \equiv \mathbb{N}_{1}\left(\begin{array}{l}
1 \\
1
\end{array}\right)+\mathbb{N}_{0}\left(\begin{array}{l}
0 \\
1
\end{array}\right), \\
& V_{0} \equiv \mathbb{N}_{0}\left(\begin{array}{l}
1 \\
0
\end{array}\right) .
\end{aligned}
$$

Their explicit values are given by,

$$
\begin{aligned}
& V_{5}=D\left(\begin{array}{l}
0 \\
2
\end{array}\right), \\
& V_{4}=D\left(\begin{array}{c}
2 \\
16+6 \xi
\end{array}\right), \\
& V_{3}=D\left(\begin{array}{c}
16+4 \xi \\
-4 \ell^{2}-4 \ell+32 \xi+8-\xi \ell^{2}-\xi \ell+6 \xi^{2}
\end{array}\right), \\
& V_{2}=D\left(\begin{array}{c}
-4 \ell^{2}-4 \ell+20 \xi+24-\xi \ell^{2}-\xi \ell+2 \xi^{2} \\
-8 \xi \ell^{2}-8 \xi \ell-4 \xi-48+22 \xi^{2}-2 \xi^{2} \ell^{2}-2 \xi^{2} \ell+2 \xi^{3}
\end{array}\right), \\
& V_{1}=D\left(\begin{array}{c}
(\xi+2)\left(6 \xi^{2}-\xi^{2} \ell^{2}-\xi^{2} \ell-\xi \ell^{2}-18 \xi-\xi \ell+\ell^{4}+11 \ell^{2}+2 \ell^{3}+10 \ell\right)
\end{array}\right), \\
& V_{0}=D\left(\begin{array}{c}
\ell(\ell-1)(\ell+2)(\ell+1)(\xi+2) \\
\ell(\xi+2)(\xi-4)(\ell-1)(\ell+2)(\ell+1)
\end{array}\right) \cdot
\end{aligned}
$$

Equation 4.9 is for a column vector, and can be regarded as two scalar differential equations that we refer to as the "upper" and the "lower". The upper ODE is of the fourth order, while the lower ODE is of fifth order. Not surprisingly, the lower equation is the first derivative of the upper equation, provided that $F^{\alpha}(\boldsymbol{r})$ is divergence free. Hence the two equations are dependent, and we restrict our attention to the upper equation. To simplify it, we divide both sides by $D r^{\xi}$, replace $d_{1}(r)$ by $\psi(r)$ and define the RHS to be the function $S(r)$,

$$
S(r) \equiv D^{-1} r^{-\xi} \rho_{1}^{*}(r) .
$$

After doing so, we reach the following equation:

$$
\psi^{(4)}+a_{3} \frac{\psi^{(3)}}{r}+a_{2} \frac{\psi^{(2)}}{r^{2}}+a_{1} \frac{\psi^{(1)}}{r^{3}}+a_{0} \frac{\psi}{r^{4}}=S(r) .
$$

Its homogeneous solution is easily found once we substitute,

$$
\psi(r)=\psi_{0} r^{\zeta}
$$


The scaling exponents are the roots of the polynomial,

$$
\begin{aligned}
P(\zeta) & =\zeta(\zeta-1)(\zeta-2)(\zeta-3) \\
& +a_{3} \zeta(\zeta-1)(\zeta-2)+a_{2} \zeta(\zeta-1)+a_{1} \zeta+a_{0}
\end{aligned}
$$

and are found to be real and nondegenerate. Two of them are positive while the other two are negative, given in a decreasing order by

$$
\begin{aligned}
& \zeta_{1}=-\frac{1}{2}-\frac{1}{2} \xi+\frac{1}{2} \sqrt{A(\ell, \xi)+\sqrt{B(\ell, \xi)}}, \\
& \zeta_{2}=-\frac{1}{2}-\frac{1}{2} \xi+\frac{1}{2} \sqrt{A(\ell, \xi)-\sqrt{B(\ell, \xi)}}, \\
& \zeta_{3}=-\frac{1}{2}-\frac{1}{2} \xi-\frac{1}{2} \sqrt{A(\ell, \xi)-\sqrt{B(\ell, \xi)}}, \\
& \zeta_{4}=-\frac{1}{2}-\frac{1}{2} \xi-\frac{1}{2} \sqrt{A(\ell, \xi)+\sqrt{B(\ell, \xi)}},
\end{aligned}
$$

where

$$
\begin{aligned}
A(\ell, \xi) \equiv & \xi^{2}+\xi \ell^{2}+\xi \ell-2 \xi+5+4 \ell+4 \ell^{2}, \\
B(\ell, \xi) \equiv & -8 \xi^{2} \ell-7 \xi^{2} \ell^{2}+16 \xi^{2}+2 \xi^{2} \ell^{3}+\xi^{2} \ell^{4} \\
& -8 \xi \ell^{2}-8 \xi \ell-32 \xi+16+64 \ell+64 \ell^{2} .
\end{aligned}
$$

In the limit $\xi \rightarrow 0$ the roots become:

$$
\begin{aligned}
& \zeta_{1}=\ell+1, \\
& \zeta_{2}=\ell-1,
\end{aligned}
$$
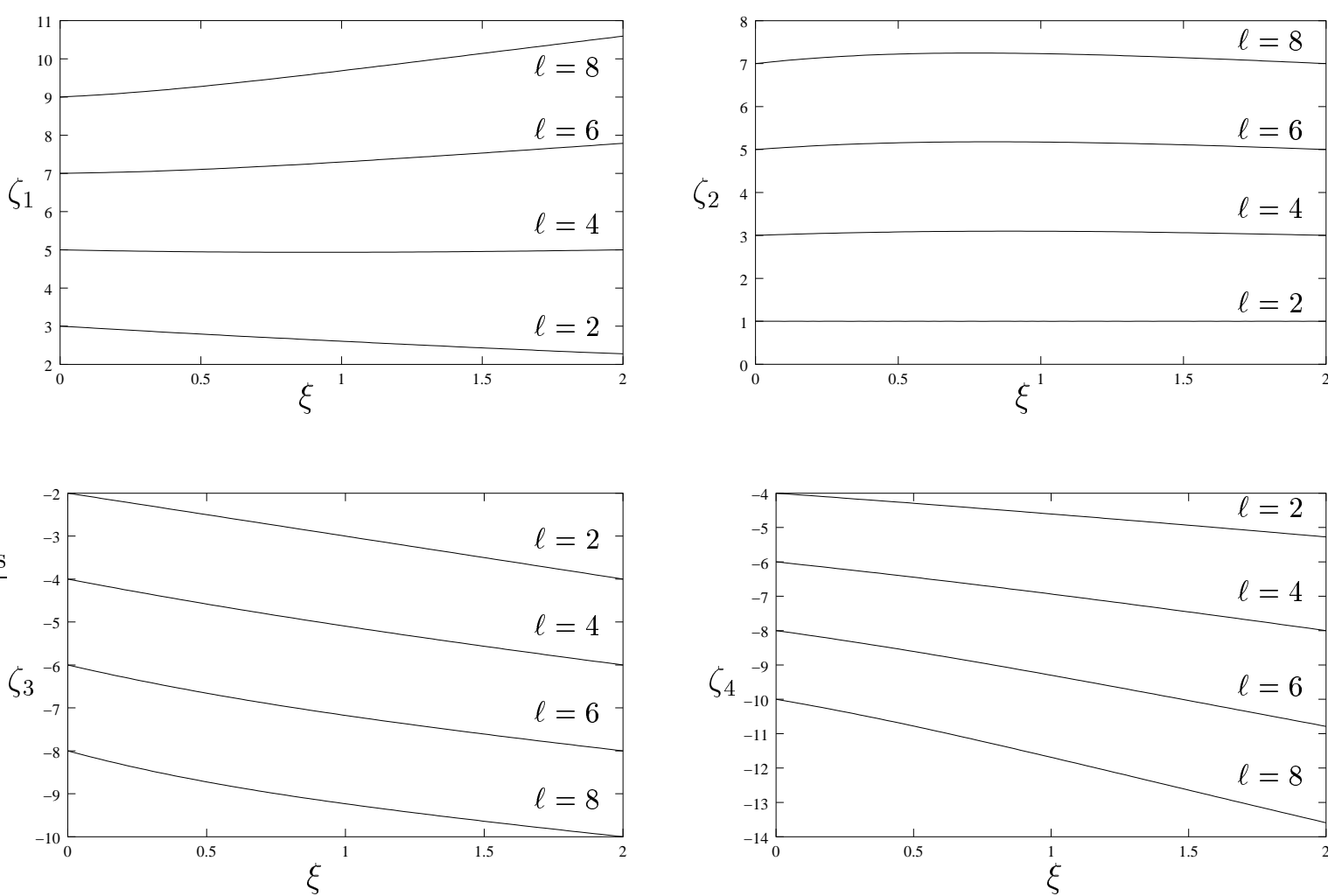

$$
\begin{aligned}
& \zeta_{3}=-\ell, \\
& \zeta_{4}=-\ell-2 .
\end{aligned}
$$

Figure 1 displays the first few exponents as a function of $\xi$. We note that the spectrum has no sign of saturation as $\ell$ increases. Before we discuss the meaning of this observation we will make sure that these solutions are physically relevant and participate in the full (exact) solution including boundary conditions.

The general solution of Eq. (4.13) is traditionally given as the sum of a special solution of the non-homogeneous equation plus a linear combination of the zero modes. However, when attempting to match the solution to the boundary conditions, it is more convenient to represent it as

$$
\psi(r)=\sum_{i=1}^{4} \underbrace{\frac{r^{\zeta_{i}}}{\left(\zeta_{i}-\zeta_{1}\right) \cdots\left(\zeta_{i}-\zeta_{4}\right)}}_{\text {all different roots }} \int_{m_{i}}^{r} d x x^{3-\zeta_{i}} S(x),
$$

where the free parameters of the solution are the four constants $m_{i}$. Indeed a change in $m_{i}$ is equivalent to adding to the solution a const $\times r^{\zeta_{i}}$. In the next subsection we find the values of $m_{i}$ that match the boundary conditions, and discuss the properties of the solution.

FIG. 1. Scaling exponents of the first few $\ell$ 's as functions of $\xi$ 


\section{B. Boundary conditions and inertial-range behavior}

From Eq. (4.20) it is clear that the only values of $m_{i}$ that guarantee that the solution remains finite as $r \rightarrow 0$ and that it decays as $r \rightarrow \infty$ are $m_{1}=m_{2}=+\infty$, $m_{3}=m_{4}=0$

$$
\begin{aligned}
& \psi(r)=-\frac{r^{\zeta_{1}}}{\left(\zeta_{1}-\zeta_{2}\right)\left(\zeta_{1}-\zeta_{3}\right)\left(\zeta_{1}-\zeta_{4}\right)} \int_{r}^{\infty} d x x^{3-\zeta_{1}} S(x) \\
& -\frac{r^{\zeta_{2}}}{\left(\zeta_{2}-\zeta_{1}\right)\left(\zeta_{2}-\zeta_{3}\right)\left(\zeta_{2}-\zeta_{4}\right)} \int_{r}^{\infty} d x x^{3-\zeta_{2}} S(x) \\
& +\frac{r^{\zeta_{3}}}{\left(\zeta_{3}-\zeta_{1}\right)\left(\zeta_{3}-\zeta_{2}\right)\left(\zeta_{3}-\zeta_{4}\right)} \int_{0}^{r} d x x^{3-\zeta_{3}} S(x) \\
& +\frac{r^{\zeta_{4}}}{\left(\zeta_{4}-\zeta_{1}\right)\left(\zeta_{4}-\zeta_{2}\right)\left(\zeta_{4}-\zeta_{3}\right)} \int_{0}^{r} d x x^{3-\zeta_{4}} S(x) .
\end{aligned}
$$

To understand the asymptotics of this solution we find from Eq. (4.12) that for $x \ll L, S(x)$ has a leading term that goes like $x^{\ell-1-\xi}$, whereas for for $x \gg L, S(x)$ decays rapidly. It is now straightforward to prove that for $r \ll L$, the $\zeta_{3}$ and $\zeta_{4}$ terms scale like $r^{\ell+3-\xi}$, the $\zeta_{2}$ term scales like $r^{\zeta_{2}}$, and the $\zeta_{1}$ term scales like $r^{\zeta_{1}}$ for values of $\xi$ for which $\zeta_{1}<\ell+3-\xi$ and like $r^{\ell+3-\xi}$ otherwise. In addition, it is easy to see that for $r \gg L, \psi(r)$ exhibits an algebraic decay: the $\zeta_{1}$ and $\zeta_{2}$ terms decay rapidly due to the decay of $S(x)$ whereas the $\zeta_{3}$ and $\zeta_{4}$ terms decay algebraicaly like $r^{\zeta_{i}}$, respectively. The asymptotics of the full solution are thus given by

$$
\psi(r) \sim\left\{\begin{array}{ll}
r^{\zeta_{2}}, & r \ll L \\
r^{\zeta_{3}}, & r \gg L
\end{array} .\right.
$$

The obvious conclusion is that there is no saturation in the anisotropic scaling exponents as $\ell$ increases. The lack of contradiction with the existence of an integral over all space has two aspects. The main one is simple and obvious. The integro-differential equation (3.1) for $C^{\alpha}$ has a differential version (3.6). Solving the differential version, we are unaffected by any considerations of convergence of integrals and therefore the solution may contain exponents that increase with $\ell$ without limit. Nevertheless, the full solution 4.21) exhibits a crossover at $L$ : it increases in the inertial range $r \ll L$ and decays for $r \gg L$. Thus plugging it back to the integro-differential equation we are guaranteed that no divergence occurs.

The question why the cross-over length $L$ does not spoil the scale invariance in the intertial range still remains. The answer is found in differential form of the equation of motion, given by Eq. (3.6). From this equation we find that the integrand is a Green's function times a Laplacian of a tensor. By definition, such an integral localizes, i.e., it is fully determined by the value of the tensor at the external vector $\boldsymbol{r}$. In the language of Eq. (1.1) $A(\boldsymbol{y})=\nabla^{2} B(\boldsymbol{y})$

The second and less obvious aspect is that the window of locality widens up with $\ell$. This is due to the cancellations in the angular integration of the anisotropic solutions that are due to the orthogonality of the $Y_{\ell m}(\hat{\boldsymbol{r}})$ and their generalizations $B_{q \ell m}^{\alpha}(\hat{\boldsymbol{r}})$. To demonstrate this consider again the simple integral (1.1), and assume that $C(\boldsymbol{y})$ belongs to $(\ell, m)$ sector, i.e.,

$$
C(\boldsymbol{y})=a(y) Y_{\ell m}(\hat{\boldsymbol{y}}) .
$$

For $y \gg r$, we may expand the Green function in $r / y$,

$$
\begin{aligned}
G(\boldsymbol{r}-\boldsymbol{y}) & =-\frac{1}{4 \pi|\boldsymbol{r}-\boldsymbol{y}|} \\
& =-\frac{1}{4 \pi y} \sum_{n=0}^{\infty} a_{n}\left[\left(\frac{r}{y}\right)^{2}-2 \frac{\boldsymbol{r} \cdot \hat{\boldsymbol{y}}}{y}\right]^{n} .
\end{aligned}
$$

Here $a_{n}$ are Taylor coefficients. Obviously the dangerous terms for the infrared convergence are those with low values of $n$. However, all these terms will vanish for $n<\ell$ in the angular integration against $Y_{\ell m}(\hat{\boldsymbol{y}})$. The reason is that all these terms are of the form $r^{n_{1}} y^{n_{2}}(\boldsymbol{r} \cdot \hat{\boldsymbol{y}})^{n_{3}}$ with $n_{3}<\ell$. The angular part here has projections only $Y_{\ell^{\prime} m^{\prime}}$ with $\ell^{\prime} \leq k_{3}<\ell$. The first term to contribute comes when $n=\ell$, and is proportional to the amplitude integral $\int_{r}^{\infty} d y y^{2} a_{\ell m}(y) y^{-\ell-1}$. For a power law $a_{\ell m}(y) \sim y^{\lambda}$ this implies locality for

$$
\lambda<\ell-2
$$

instead of $\lambda<-2$, as in the isotropic sector. The lower bound of the window of locality is also extended and a similar analysis for $y \ll r$ leads to $\lambda>-\ell-3$. For the toy model this translates to the window of locality

$$
-\ell-\xi<\zeta_{i}<\ell+1-\xi \text {. }
$$

From the previous analysis we find that the leading power law of the full solution in the inertial range is $r^{\zeta_{2}}$, which is inside this "extended" window of locality. Nevertheless, the subleading power $r^{\zeta_{1}}$ originating from the first term in Eq. (4.21) is above this window, and its presence in the solution can be explained only using the first mechanism.

We will see when we turn back to the linear pressure model that both these mechanisms operate there as well, leading again to a lack of saturation in the exponents.

\section{SOLVING THE LINEAR PRESSURE MODEL}

We now return to the linear pressure model. As mentioned before in Sec. IIB, we see no way of eliminating all integrals from the equation and therefore we will not look for a full solution. Nevertheless, we shall be able to calculate the zero modes and hence the scaling exponents. Our strategy relies heavily on the conclusions of the last section: we will apply two Laplacians to the equation for $C^{\alpha \beta}(\boldsymbol{r})$ in order to eliminate the integrals of the two projection operators $\hat{\mathcal{P}}_{\mathrm{L}}$ and $\hat{\mathcal{P}}_{\mathrm{R}}$. The resulting equation will still contain the nontrivial integral. Using 
numerical integration we will solve the homogeneous part of this equation, i.e., we shall find its zero modes. These are scale invariant solutions that solve an equation containing an integral. Their exponent must therefore lie within the "extended" ( $\ell$ dependent) window of locality. Finally, we will argue that these zero modes are a part of the full solution that decays for $r \gg L$, and therefore solve the original equation as well.

\section{A. Equations for the zero modes}

We start from Eqs. 2.24) and (2.25). In appendix B we perform integration by parts and algebraic manipulations to bring the nontrivial integral in Eq. (2.25) to a more tractable form. The result of this process is

$$
\begin{aligned}
& T^{\alpha \beta}(\boldsymbol{r})=-\frac{1}{2} \hat{\mathcal{P}}_{\mathrm{L}} K^{\mu \nu} \partial_{\mu} \partial_{\nu} C^{\alpha \beta}(\boldsymbol{r}) \\
& -\frac{1}{2} \frac{12 \xi D}{(\xi-3)(\xi-5)} \int d \boldsymbol{y} G(\boldsymbol{y}) y^{\xi-2} \partial^{2} C^{\alpha \beta}(\boldsymbol{r}-\boldsymbol{y})
\end{aligned}
$$

which is true for every $\xi \neq 1$. The $\xi=1$ case will not be treated here explicitly. Nevertheless, we will argue $a$ posteriori that the results for $\xi=1$ can be deduced from the $\xi \neq 1$ results by continuity.

Looking at Eq. (5.1), we note that when $\xi=2$, the integral on the RHS of the above equation trivializes to a local term $C^{\alpha \beta}(\boldsymbol{r})$. In this limiting case the model can be fully solved utilizing the same machinery used in the previous section. The solution can then be used to check the zero modes computed below for arbitrary values of $\xi$.

To proceed, we substitute Eq. (5.1) into Eq. (2.24), noting that the projector $\hat{\mathcal{P}}_{\mathrm{R}}$ leaves the nontrivial integral in Eq. (5.1) invariant since it is divergence-free in both indices. Setting $\partial_{t} C^{\alpha \beta}(\boldsymbol{r}, t)=0$ in the stationary case, we arrive at the following equation:

$$
\begin{aligned}
& 0=-\left[\hat{\mathcal{P}}_{\mathrm{R}} \hat{\mathcal{P}}_{\mathrm{L}} K^{\mu \nu} \partial_{\mu} \partial_{\nu} C^{\alpha \beta}\right](\boldsymbol{r}) \\
& -\frac{12 \xi D}{(\xi-3)(\xi-5)} \int d \boldsymbol{y} G(\boldsymbol{y}) y^{\xi-2} \partial^{2} C^{\alpha \beta}(\boldsymbol{r}-\boldsymbol{y}) \\
& +2 \kappa \partial^{2} C^{\alpha \beta}(\boldsymbol{r})+F^{\alpha \beta}(\boldsymbol{r}) .
\end{aligned}
$$

As in the toy model, we apply two Laplacians to the above equation in order to get rid of the integrals of the projection operators and obtain

$$
\begin{aligned}
& 0=-\partial^{4}\left[\hat{\mathcal{P}}_{\mathrm{R}} \hat{\mathcal{P}}_{\mathrm{L}} K^{\mu \nu} \partial_{\mu} \partial_{\nu} C^{\alpha \beta}\right](\boldsymbol{r}) \\
& -\frac{12 \xi D}{(\xi-3)(\xi-5)} \int d \boldsymbol{y} G(\boldsymbol{y}) y^{\xi-2} \partial^{6} C^{\alpha \beta}(\boldsymbol{r}-\boldsymbol{y}) \\
& +2 \kappa \partial^{6} C^{\alpha \beta}(\boldsymbol{r})+\partial^{4} F^{\alpha \beta}(\boldsymbol{r}) .
\end{aligned}
$$

Here and in the following, the operator $\partial^{2 n}$ should be interpreted as $\left(\partial^{2}\right)^{n}$. We now seek the homogeneous stationary solutions of $C^{\alpha \beta}(\boldsymbol{r})$ in the inertial range (zero modes). These satisfy the equations obtained by neglecting the dissipation and setting the forcing and time derivative to zero,

$$
\begin{aligned}
0 & =\partial^{4} K^{\mu \nu} \partial_{\mu} \partial_{\nu} C^{\alpha \beta}(\boldsymbol{r})+\partial^{\alpha} \partial^{\beta} \partial_{\tau} \partial_{\sigma} K^{\mu \nu} \partial_{\mu} \partial_{\nu} C^{\tau \sigma}(\boldsymbol{r}) \\
& -\partial^{\alpha} \partial_{\tau} \partial^{2} K^{\mu \nu} \partial_{\mu} \partial_{\nu} C^{\tau \beta}(\boldsymbol{r})-\partial^{\beta} \partial_{\tau} \partial^{2} K^{\mu \nu} \partial_{\mu} \partial_{\nu} C^{\alpha \tau}(\boldsymbol{r}) \\
& +\frac{12 \xi D}{(\xi-3)(\xi-5)} \int d \boldsymbol{y} G(\boldsymbol{y}) y^{\xi-2} \partial^{6} C^{\alpha \beta}(\boldsymbol{r}-\boldsymbol{y})
\end{aligned}
$$

Let us now define the RHS of the above equation as the "zero-modes operator" $\hat{\mathcal{O}}(\xi)$ and write the zero-modes equation compactly as

$$
0=\left[\hat{\mathcal{O}}(\xi) C^{\alpha \beta}\right](\boldsymbol{r})
$$

To solve it, we write the solution $C^{\alpha \beta}(\boldsymbol{r})$ in a basis that diagonalizes $\hat{\mathcal{O}}(\xi)$. This is done in the next subsection.

\section{B. The $\mathbf{S O}(3)$ decomposition}

To diagonalize $\hat{\mathcal{O}}(\xi)$ we must look for its symmetries by looking for the operations that commute with it. From Eq. (5.4) it is easy to see that these are rotations, scaling, permutation of indices, and flipping of $\boldsymbol{r}$. As a result $\hat{\mathcal{O}}(\xi)$ is block diagonalized by tensors $C^{\alpha \beta}(\boldsymbol{r})$ that have the following properties:

- They belong to a definite sector $(\ell, m)$ of the $\mathrm{SO}(3)$ group.

- They have a definite scaling behavior, i.e., are proportional to $r^{\lambda}$ with some scaling exponent $\lambda$.

- They are either symmetric or antisymmetric under permutations of indices.

- They are either even or odd in $\boldsymbol{r}$.

In 81 we discuss these types of tensors in detail. Here we only quote the final results. In every sector $(\ell, m)$ of the rotation group with $\ell>1$, one can find nine independent tensors $X^{\alpha \beta}(\boldsymbol{r})$ that scale like $r^{\lambda}$. They are given by $r^{\lambda} B_{\ell m, q}^{\alpha \beta}(\hat{\boldsymbol{r}})$ where the index $q$ runs from 1 to 9 enumerating the different spherical tensors. These nine tensors can be further subdivided into four subsets:

- Subset I of symmetric tensors with $(-)^{\ell}$ parity, containing 4 tensors.

- Subset II of symmetric tensors with $(-)^{\ell+1}$ parity, containing 2 tensors.

- Subset III of antisymmetric tensors with $(-)^{\ell+1}$ parity, containing 2 tensors.

- Subset IV of symmetric tensors with $(-)^{\ell}$ parity, containing 1 tensor. 
Due to the diagonalization of $\hat{\mathcal{O}}(\xi)$ by these subsets, the equation for the zero modes foliates and we can compute the zero modes in each subset separately. In this paper, we choose to focus on subset I, which has the richest structure. The four tensors in this subset are given by

$$
\begin{aligned}
& B_{1, \ell m}^{\alpha \beta}(\hat{\boldsymbol{r}})=r^{-\ell-2} r^{\alpha} r^{\beta} \Phi_{\ell m}(\boldsymbol{r}) \\
& B_{2, \ell m}^{\alpha \beta}(\hat{\boldsymbol{r}})=r^{-\ell}\left[r^{\alpha} \partial^{\beta}+r^{\beta} \partial^{\alpha}\right] \Phi_{\ell m}(\boldsymbol{r}), \\
& B_{3, \ell m}^{\alpha \beta}(\hat{\boldsymbol{r}})=r^{-\ell} \delta^{\alpha \beta} \Phi_{\ell m}(\boldsymbol{r}) \\
& B_{4, \ell m}^{\alpha \beta}(\hat{\boldsymbol{r}})=r^{-\ell+2} \partial^{\alpha} \partial^{\beta} \Phi_{\ell m}(\boldsymbol{r}) .
\end{aligned}
$$

We expect the calculation of the other subsets to be easier once one is familiar with the techniques we are about to develop. Finally. we note that since the correlation $C^{\alpha \beta}(\boldsymbol{r})$ has to fulfill $C^{\alpha \beta}(\boldsymbol{r})=C^{\beta \alpha}(-\boldsymbol{r})$, our subset I solution will be valid only for even $\ell$ s.

Expanding $C^{\alpha \beta}(\boldsymbol{r})$ in subset I,

$$
\begin{aligned}
C^{\alpha \beta}(\boldsymbol{x})= & r^{\lambda}\left[c_{1} B_{1, \ell m}^{\alpha \beta}(\hat{\boldsymbol{r}})+c_{2} B_{2, \ell m}^{\alpha \beta}(\hat{\boldsymbol{r}})\right. \\
& \left.+c_{3} B_{3, \ell m}^{\alpha \beta}(\hat{\boldsymbol{r}})+c_{4} B_{4, \ell m}^{\alpha \beta}(\hat{\boldsymbol{r}})\right],
\end{aligned}
$$

and plugging it back into PDE (5.4), we obtain a linear equation for the coefficients $c_{1}, c_{2}, c_{3}, c_{4}$ that depend on the parameters $\lambda, \ell, \xi$. In the four-dimensional space of column vectors $\left(c_{1}, c_{2}, c_{3}, c_{4}\right)$, we can write it as

$$
(\mathbb{O}(\lambda ; \ell, \xi) \quad)\left(\begin{array}{l}
c_{1} \\
c_{2} \\
c_{3} \\
c_{4}
\end{array}\right)=0
$$

where $\mathbb{O}(\lambda ; \ell, \xi)$ is a $4 \times 4$ matrix that represents the zeromodes operator $\hat{\mathcal{O}}(\xi)$.

To continue, we note that due to the incompressibility constraint $(2.20)$ of $C^{\alpha \beta}(\boldsymbol{r})$, not all vectors $\left(c_{1}, c_{2}, c_{3}, c_{4}\right)$ are allowed; for a given $\lambda, \ell$, only certain combinations of the $B_{\ell m, q}^{\alpha \beta}(\hat{\boldsymbol{r}})$ lead to a divergence free $C^{\alpha \beta}(\boldsymbol{r})$. A simple calculation [1] shows that these belong to a twodimensional subspace, which is spanned by the "incompressible vectors"

$$
\begin{aligned}
& \left|u_{1}(\lambda ; \ell)\right\rangle=\left(\begin{array}{c}
-\ell(\lambda-\ell) \\
(\lambda+2)(\ell-1)(\lambda-\ell+2) \\
0 \\
-(\lambda+3) \\
\lambda-\ell \\
0 \\
1
\end{array}\right), \\
& \left|u_{2}(\lambda ; \ell)\right\rangle=\left(\begin{array}{c}
(\lambda-\ell+2) \\
(\lambda+2)(\ell-1)
\end{array}\right) .
\end{aligned}
$$

The zero modes exponents $\lambda$ can be found by requiring the equation

$$
\mathbb{O}(\lambda ; \ell, \xi)\left[a_{1}\left|u_{1}(\lambda ; \ell)\right\rangle+a_{2}\left|u_{2}(\lambda ; \ell)\right\rangle\right]=0
$$

to admit a nontrivial solution. The explicit computation of the matrix $\mathbb{O}(\lambda ; \ell, \xi)$ is technically very cumbersome due to the presence of the integral term. We shall therefore use an implicit method to determine whether Eq. (5.10) has a nontrivial solution or not. The basic idea is that the calculation of the nontrivial integral in Eq. (5.4) can be simplified if we contract its free indices with the two isotropic tensors $\hat{r}_{\alpha} \hat{r}_{\beta}$ and $\delta_{\alpha \beta}$. Therefore, instead of solving Eq. (5.4) explicitly, we will contract its RHS with these two tensors and require that the two resultant scalars vanish simultaneously. Obviously this would provide us with a necessary condition for the solvability of Eq. (5.4). Nevertheless, we shall see that it is also a sufficient condition.

Let us write the two tensors $\hat{r}_{\alpha} \hat{r}_{\beta}$ and $\delta_{\alpha \beta}$ in matrix notation as two row vectors $\left\langle w_{1}(\ell)\right|,\left\langle w_{2}(\ell)\right|$ given by

$$
\begin{aligned}
& \left\langle w_{1}(\ell)\right| \equiv\left(\begin{array}{llll}
1 & 2 \ell & 1 & \ell(\ell-1)
\end{array}\right) \text { contraction with } \hat{r}_{\alpha} \hat{r}_{\beta} \\
& \left\langle w_{2}(\ell)\right| \equiv\left(\begin{array}{llll}
1 & 2 \ell & 3 & 0
\end{array}\right) \quad \text { contraction with } \delta_{\alpha \beta} .
\end{aligned}
$$

The contraction of $\delta_{\alpha \beta}$ and $\hat{r}_{\alpha} \hat{r}_{\beta}$ with another tensor is translated to the usual matrix multiplication of these row vectors with a column vector. For example, if $|c\rangle$ is a column vector whose components are given by $c_{i}, i=1, \ldots 4$, then

$$
\begin{aligned}
\delta_{\alpha \beta} & \left(c_{1} B_{1, \ell m}^{\alpha \beta}(\hat{\boldsymbol{r}})+c_{2} B_{2, \ell m}^{\alpha \beta}(\hat{\boldsymbol{r}})+c_{3} B_{3, \ell m}^{\alpha \beta}(\hat{\boldsymbol{r}})+c_{4} B_{4, \ell m}^{\alpha \beta}(\hat{\boldsymbol{r}})\right) \\
& =\left(c_{1}+2 \ell c_{2}+3 c_{3}\right) Y_{\ell m}(\hat{\boldsymbol{r}}) \\
& =\left\langle w_{2}(\ell) \mid c\right\rangle Y_{\ell m}(\hat{\boldsymbol{r}}) .
\end{aligned}
$$

Returning to the zero-mode equations, we now define the $2 \times 2$ "reduced matrix" $O_{i j}(\lambda ; \ell, \xi)$ by

$$
O_{i j}(\lambda ; \ell, \xi) \equiv\left\langle w_{i}(\ell)|\mathbb{O}(\lambda ; \ell, \xi)| u_{j}(\lambda ; \ell)\right\rangle
$$

Obviously, the existence of a nontrivial solution that makes the two contractions vanish is equivalent to the requirement that $O_{i j}(\lambda ; \ell, \xi)$ is singular, i.e.,

$$
\operatorname{det} O_{i j}(\lambda ; \ell, \xi)=0
$$

The above condition is also sufficient for the solvability of the zero-modes equation. To see that, notice that the RHS of Eq. (5.2) and therefore the RHS of Eq. (5.4) produce tensors that are divergence-free in both indices. Thus the vectors $\mathbb{O}(\lambda ; \ell, \xi)\left|u_{i}(\lambda ; \ell)\right\rangle$ will belong to the two-dimensional subspace that is spanned by $\left|u_{j}(\lambda+\xi-6 ; \ell)\right\rangle$. Since the transformation matrix

$$
U_{i j} \equiv\left\langle w_{i}(\ell) \mid u_{j}(\lambda+\xi-6 ; \ell)\right\rangle \text {, }
$$


is nonsingular for all values of $\lambda$ and $\xi$, we find that $\mathbb{O}(\lambda ; \ell, \xi)\left|u_{1}(\lambda ; \ell)\right\rangle$ and $\mathbb{O}(\lambda ; \ell, \xi)\left|u_{2}(\lambda ; \ell)\right\rangle$ are linearly dependent if and only if Eq. (5.14) holds.

Looking at Eq. (5.4), we recognize that $\hat{\mathcal{O}}(\xi)$ is a sum of a differential and an integral operator. Consequently, $\mathbb{O}(\lambda ; \ell, \xi)$ and $O_{i j}(\lambda ; \ell, \xi)$ can be written as a sum of cor- responding parts,

$$
\begin{aligned}
\mathbb{O}(\lambda ; \ell, \xi) & =\mathbb{O}^{d i f}(\lambda ; \ell, \xi)+\mathbb{O}^{i n t}(\lambda ; \ell, \xi), \\
O_{i j}(\lambda ; \ell, \xi) & =O_{i j}^{d i f}(\lambda ; \ell, \xi)+O_{i j}^{i n t}(\lambda ; \ell, \xi) .
\end{aligned}
$$

These parts are of a different nature and thus we dedicate two different subsections for their calculation.

\section{Form of the differential operator}

The calculation of $O_{i j}^{d i f}(\lambda ; \ell, \xi)$ can be done directly by calculating the matrix $\mathbb{O}^{d i f}(\lambda ; \ell, \xi)$, employing the same techniques that are used in Refs [1,8] and in sec. [II]. Here we merely give the mid and final results.

From Eq. (5.4), we see that the differential part of the zero-mode operator is given by

$$
\begin{aligned}
\hat{\mathcal{O}}^{d i f}(\xi) C^{\alpha \beta} \equiv & \partial^{2} \partial^{2} K^{\mu \nu} \partial_{\mu} \partial_{\nu} C^{\alpha \beta}+\partial^{\alpha} \partial^{\beta} \partial_{\tau} \partial_{\sigma} K^{\mu \nu} \partial_{\mu} \partial_{\nu} C^{\tau \sigma} \\
& -\partial^{\alpha} \partial_{\tau} \partial^{2} K^{\mu \nu} \partial_{\mu} \partial_{\nu} C^{\tau \beta}-\partial^{\beta} \partial_{\tau} \partial^{2} K^{\mu \nu} \partial_{\mu} \partial_{\nu} C^{\alpha \tau}
\end{aligned}
$$

To find its matrix representation it is convenient to first calculate following operators:

- $\mathbb{L}(\lambda ; \ell): X^{\alpha \beta} \longrightarrow \partial^{2} X^{\alpha \beta}$

$$
\mathbb{L}(\lambda ; \ell)=\left(\begin{array}{llll}
(\lambda-\ell-2)(\lambda+j+3) & 0 & 0 & 0 \\
2 & (\lambda-\ell)(\lambda+\ell+1) & 0 & 0 \\
2 & 0 & (\lambda-\ell)(\lambda+\ell+1) & 0 \\
0 & 4 & 0 & (\lambda-\ell+2)(\lambda+\ell-1)
\end{array}\right),
$$

- $\mathbb{M}_{1}(\lambda ; \ell): X^{\alpha \beta} \longrightarrow \partial^{\alpha} \partial^{\beta} \partial_{\tau} \partial_{\sigma} X^{\tau \sigma}$

$$
\mathbb{M}_{1}(\lambda ; \ell)=\left(\begin{array}{c}
(\lambda-\ell-2)(\lambda-\ell-4) \\
\lambda-\ell-2 \\
\lambda-\ell-2 \\
1
\end{array}\right) \times\left(\begin{array}{c}
(\lambda+1)(\lambda+2) \\
2 \ell(\lambda+2)(\lambda-\ell) \\
(\lambda+1+\ell)(\lambda-\ell) \\
\ell(\lambda-\ell)(\ell-1)(\lambda-\ell+2)
\end{array}\right)^{\mathrm{T}}
$$

- $\mathbb{M}_{2}(\lambda ; \ell): X^{\alpha \beta} \longrightarrow \partial^{\alpha} \partial_{\tau} X^{\tau \beta}+\partial^{\beta} \partial_{\tau} X^{\alpha \tau}$

$$
\mathbb{M}_{2}(\lambda ; \ell)=\left(\begin{array}{llll}
2(\lambda-\ell-2)(\lambda+2) & 2 \ell(\lambda-\ell)(\lambda-\ell-2) & 2(\lambda-\ell-2)(\lambda-\ell) & 0 \\
\lambda+2 & (\lambda-\ell)(\ell+\lambda+3) & 2(\lambda-\ell) & (\lambda-\ell)(\ell-1)(\lambda-\ell+2) \\
2(\lambda+2) & 2 \ell(\lambda-\ell) & 2(\lambda-\ell) & 0 \\
0 & 2(\lambda+3) & 2 & 2(\ell-1)(\lambda-\ell+2)
\end{array}\right)
$$

- $\mathbb{K}(\lambda ; \ell, \xi): X^{\alpha \beta} \longrightarrow K^{\mu \nu}(\xi) \partial_{\mu} \partial_{\nu} X^{\alpha \beta}$

$$
\mathbb{K}(\lambda ; \ell, \xi)=D[(\xi+2) \mathbb{L}(\lambda ; \ell)-\xi \lambda(\lambda-1) \mathbb{1}]
$$

With these four matrices, the matrix form of the differential part is written compactly as

$$
\begin{aligned}
& \mathbb{O}^{d i f}(\lambda ; \ell, \xi)=[\mathbb{L}(\lambda+\xi-4 ; \ell) \mathbb{L}(\lambda+\xi-2 ; \ell) \\
& \quad+\mathbb{M}_{1}(\lambda+\xi-2 ; \ell)
\end{aligned}
$$




$$
\left.-\mathbb{M}_{2}(\lambda+\xi-4 ; \ell) \mathbb{L}(\lambda+\xi-2 ; \ell)\right] \mathbb{K}(\lambda ; \ell, \xi) .
$$

$O_{i j}^{d i f}(\lambda ; \ell, \xi)$ is then computed directly from definition.

\section{Form of the integral operator} by

The integral part of the zero-modes operator is given

$$
\begin{aligned}
& {\left[\hat{\mathcal{O}}^{i n t}(\xi) C^{\alpha \beta}\right](\boldsymbol{r}) \equiv} \\
& \frac{12 \xi D}{(\xi-3)(\xi-5)} \int d \boldsymbol{y} G(\boldsymbol{r}-\boldsymbol{y})|\boldsymbol{r}-\boldsymbol{y}|^{\xi-2} \partial^{6} C^{\alpha \beta}(\boldsymbol{y})
\end{aligned}
$$

To calculate the reduced matrix (5.13), we have to compute the contraction of $\hat{\mathcal{O}}^{i n t}(\xi) C^{\alpha \beta}$ with $\hat{r}_{\alpha} \hat{r}_{\beta}$ and $\delta_{\alpha \beta}$. To do that, let the tensor $X^{\alpha \beta}(\boldsymbol{r})$ be of the form of the trial solution (5.7), i.e., let it belong to the $(\ell, m)$ sector of $\mathrm{SO}(3)$, be divergence free in both indices, and proportional to $r^{\lambda}$. If we denote in matrix notation $X^{\alpha \beta}(\boldsymbol{r})$ by the column vector $|x\rangle$, then from the isotropy of the tensors $\hat{r}_{\alpha} \hat{r}_{\beta}, \delta_{\alpha \beta}$, we have the following useful identities:

$$
\begin{aligned}
\hat{r}_{\alpha} \hat{r}_{\beta} X^{\alpha \beta}(\boldsymbol{r}) & =\left\langle w_{1}(\ell) \mid x\right\rangle r^{\lambda} Y_{\ell m}(\hat{\boldsymbol{r}}), \\
\delta_{\alpha \beta} X^{\alpha \beta}(\boldsymbol{r}) & =\left\langle w_{2}(\ell) \mid x\right\rangle r^{\lambda} Y_{\ell m}(\hat{\boldsymbol{r}}) .
\end{aligned}
$$

Consider now the contraction of

$$
\int d \boldsymbol{y} G(\boldsymbol{r}-\boldsymbol{y})|\boldsymbol{r}-\boldsymbol{y}|^{\xi-2} X^{\alpha \beta}(\boldsymbol{y}),
$$

with $\delta_{\alpha \beta}$

$$
\begin{aligned}
I_{1} & =\delta_{\alpha \beta} \int d \boldsymbol{y} G(\boldsymbol{r}-\boldsymbol{y})|\boldsymbol{r}-\boldsymbol{y}|^{\xi-2} X^{\alpha \beta}(\boldsymbol{y}) \\
& =\left\langle w_{2}(\ell) \mid x\right\rangle \int d \boldsymbol{y} G(\boldsymbol{r}-\boldsymbol{y})|\boldsymbol{r}-\boldsymbol{y}|^{\xi-2} y^{\lambda} Y_{\ell m}(\hat{\boldsymbol{y}})
\end{aligned}
$$

If $\lambda$ is in the window of locality of the integral above, then the integral will converge and be proportional to $r^{\lambda+\xi} Y_{\ell m}(\hat{\boldsymbol{r}})$. The scaling exponent $\lambda+\xi$ follows from power counting [remember that $G(\boldsymbol{r}) \sim 1 / r$ ] while the angular dependence is a result of the isotropy of the integration over all space. This leads us to define the proportionality factor $A(\lambda ; \xi, \ell)$,

$$
\begin{aligned}
& A(\lambda ; \ell, \xi) r^{\lambda+\xi} Y_{\ell m}(\hat{\boldsymbol{r}}) \\
& \quad \equiv \int d \boldsymbol{y} G(\boldsymbol{r}-\boldsymbol{y})|\boldsymbol{r}-\boldsymbol{y}|^{\xi-2} y^{\lambda} Y_{\ell m}(\hat{\boldsymbol{y}})
\end{aligned}
$$

with which we can write

$$
I_{1}=\left\langle w_{2}(\ell) \mid x\right\rangle A(\lambda ; \ell, \xi) r^{\lambda+\xi} Y_{\ell m}(\hat{\boldsymbol{r}}) .
$$

The prefactor $A(\lambda ; \ell, \xi)$ is the only part of the calculation that cannot be done analytically. However, instead of calculating the integral in Eq. 5.27 numerically, we can expand the integrand as a Taylor series and write $A(\lambda ; \ell, \xi)$ as an infinite series of poles in $\lambda$. This is done in detail in Appendix $\mathrm{Q}$ with the result that

$$
\begin{aligned}
& A(\lambda ; \ell, \xi)= \\
& -\frac{1}{2} \sum_{q=0}^{\infty} a_{q}(\ell, \xi)\left[\frac{1}{\lambda+3+\ell+2 q}-\frac{1}{\lambda+\xi-\ell-2 q}\right] .
\end{aligned}
$$

The coefficients $a_{q}(\ell, \xi)$ are given in Appendix C. We notice that the window of locality in the definition (5.27) of $A(\lambda ; \xi, \ell)$ can be identified from the positions of the poles in Eq. (5.29). Indeed, the boundaries of the window of locality are determined by the $q=0$ term and located at $\lambda=-\ell-3$ and $\lambda=\ell-\xi$. When $\lambda$ hits these boundaries the integral in Eq. (5.27) diverges corresponding to a pole in 5.29 . However, the above formula is valid also for values of $\lambda$ outside this window of locality for which the formal definition of $A(\lambda ; \ell, \xi)$ makes no sense. The relevance of these values to the full solution must therefore be addressed. This will be done in the next subsection where we present the values of the scaling exponents.

Let us now consider the $\hat{r}_{\alpha} \hat{r}_{\beta}$ contraction

$$
I_{2}=r^{-2} \int d \boldsymbol{y} G(\boldsymbol{r}-\boldsymbol{y})|\boldsymbol{r}-\boldsymbol{y}|^{\xi-2} r_{\alpha} r_{\beta} X^{\alpha \beta}(\boldsymbol{y})
$$

Using the identity

$$
\begin{aligned}
& r_{\alpha} r_{\beta}=\left(r_{\alpha}-y_{\alpha}\right)\left(r_{\beta}-y_{\beta}\right)+\left(r_{\alpha}-y_{\alpha}\right) y_{\beta} \\
& \quad+y_{\alpha}\left(r_{\beta}-y_{\beta}\right)+y_{\alpha} y_{\beta},
\end{aligned}
$$

we can decompose the integral into four terms:

- The $\left(r_{\alpha}-y_{\alpha}\right)\left(r_{\beta}-y_{\beta}\right)$ term:

Recalling that

$$
G(\boldsymbol{r}-\boldsymbol{y})=-\frac{1}{4 \pi|\boldsymbol{r}-\boldsymbol{y}|}
$$

it is easy to verify that

$$
\begin{aligned}
& G(\boldsymbol{r}-\boldsymbol{y})|\boldsymbol{r}-\boldsymbol{y}|^{\xi-2}\left(r_{\alpha}-y_{\alpha}\right)\left(r_{\beta}-y_{\beta}\right) \\
& \quad=\frac{1}{(\xi+1)(\xi-1)} \partial_{\alpha} \partial_{\beta} G(\boldsymbol{r}-\boldsymbol{y})|\boldsymbol{r}-\boldsymbol{y}|^{\xi+2} \\
& \quad-\frac{1}{\xi-1} G(\boldsymbol{r}-\boldsymbol{y})|\boldsymbol{r}-\boldsymbol{y}|^{\xi} \delta_{\alpha \beta}
\end{aligned}
$$

Plugging this into the integral, the term with the derivatives will vanish due to integration by parts and the divergence-free $X^{\alpha \beta}(\boldsymbol{r})$. We are therefore left with

$$
\begin{aligned}
& -\frac{r^{-2}}{\xi-1} \int d \boldsymbol{y} G(\boldsymbol{r}-\boldsymbol{y})|\boldsymbol{r}-\boldsymbol{y}|^{\xi} \delta_{\alpha \beta} X_{\ell m}^{\alpha \beta}(\boldsymbol{y}) \\
& =-\frac{r^{-2}}{\xi-1}\left\langle w_{2}(\ell) \mid x\right\rangle \int d \boldsymbol{y} G(\boldsymbol{r}-\boldsymbol{y})|\boldsymbol{r}-\boldsymbol{y}|^{\xi} y^{\lambda} Y_{\ell m}(\hat{\boldsymbol{y}})
\end{aligned}
$$


Using the identity

$$
\partial^{2} y^{\lambda+2} Y_{\ell m}(\hat{\boldsymbol{y}})=(\lambda+2-\ell)(\lambda+3+\ell) y^{\lambda} Y_{\ell m}(\hat{\boldsymbol{y}}),
$$

we further integrate by parts the last integral to finally obtain

$$
\begin{aligned}
& \frac{-\xi}{(\lambda+2-\ell)(\lambda+3+\ell)}\left\langle w_{2}(\ell) \mid x\right\rangle A(\lambda+2 ; \ell, \xi) \\
& \quad \times r^{\lambda+\xi} Y_{\ell m}(\hat{\boldsymbol{r}}) .
\end{aligned}
$$

- The $\left(r_{\alpha}-y_{\alpha}\right) y_{\beta}, y_{\alpha}\left(r_{\beta}-y_{\beta}\right)$ terms:

Using the same tricks as in the previous term, i.e., integration by parts and the fact that $X^{\alpha \beta}(\boldsymbol{y})$ is divergence free, we can easily show that both these terms vanish.
- The $y_{\alpha} y_{\beta}$ term:

Using Eq. (5.24) and the definition of $A(\lambda ; \ell, \xi)$, we directly get

$$
\left\langle w_{1}(\ell) \mid x\right\rangle A(\lambda+2 ; \ell, \xi) r^{\lambda+\xi} Y_{\ell m}(\hat{\boldsymbol{r}}) .
$$

Gathering all the terms, we see that the contraction with $\hat{r}_{\alpha} \hat{r}_{\beta}$ is equal to

$$
\begin{aligned}
I_{2}= & {\left[\left\langle w_{1}(\ell) \mid x\right\rangle-\frac{\xi}{(\lambda+2-\ell)(\lambda+3+\ell)}\left\langle w_{2}(\ell) \mid x\right\rangle\right] } \\
& \times A(\lambda+2 ; \ell, \xi) r^{\lambda+\xi} Y_{\ell m}(\hat{\boldsymbol{r}}) .
\end{aligned}
$$

To conclude, the matrix $O_{i j}^{i n t}(\lambda ; \ell, \xi)$ is given by the following equations:

$$
\begin{aligned}
\left|\tilde{u}_{i}(\lambda ; \ell, \xi)\right\rangle & =\frac{12 \xi D}{(\xi-3)(\xi-5)} \mathbb{L}(\lambda-4 ; \ell) \mathbb{L}(\lambda-2 ; \ell) \mathbb{L}(\lambda ; \ell)\left|u_{i}(\lambda ; \ell)\right\rangle, \\
O_{1, i}^{i n t}(\lambda ; \ell, \xi) & =A(\lambda-4 ; \ell, \xi)\left[\left\langle w_{1}(\ell) \mid \tilde{u}_{i}(\lambda ; \ell, \xi)\right\rangle-\frac{\xi}{(\lambda-4-\ell)(\lambda-3+\ell)}\left\langle w_{2}(\ell) \mid \tilde{u}_{i}(\lambda ; \ell, \xi)\right\rangle\right], \\
O_{2, i}^{i n t}(\lambda ; \ell, \xi) & =A(\lambda-6 ; \ell, \xi)\left\langle w_{2}(\ell) \mid \tilde{u}_{i}(\lambda ; \ell, \xi)\right\rangle .
\end{aligned}
$$

\section{Results}

Figure 2 shows the leading scaling exponents of the linear pressure model for $\ell=0,2,4,6,8,10$. The results where obtained by numerically solving Eq. (5.14) for $\xi=0,0.01,0.02, \ldots, 1.99,2$. The prefactor $A(\lambda ; \ell, \xi)$ was calculated using the formulas $(\overline{\mathrm{C} 12})$ and $(\overline{\mathrm{C} 13})$ where the infinite series of poles was truncated typically after 100 poles, when it was clear that relative contribution from the consecutive pole was smaller than the machine precision (about $10^{-14}$ ). Additionally, the numerical results were compared to the analytical results of $\xi=0,2$ and of $\ell=0$ and $\ell=2$ and found to be correct within a relative error of $10^{-10}$.

From Fig. 2, we see that in the isotropic sector and in the $\ell=2$ sector, the leading exponent is $\zeta=0$, corresponding to the trivial $C^{\alpha \beta}(\boldsymbol{r})=$ const solution. These zero modes will not contribute to the second-order structure function, which is given by

$$
S^{\alpha \beta}(\boldsymbol{r})=2\left[C^{\alpha \beta}(\boldsymbol{r})-C^{\alpha \beta}(\mathbf{0})\right]
$$

and so we have to consider the zero mode with the consecutive exponent. In the isotropic sector this exponent is exactly $\zeta=2-\xi$ as can be proven by passing to Fourier space. This special solution is a finger-print of the existence of a constant energy flux in this model. Indeed just like in Navier-Stokes turbulence, one can show analytically that the isotropic part of the triple correlation function $\left\langle v^{\alpha}(\boldsymbol{x}) w^{\mu}(\boldsymbol{x}+\boldsymbol{r}) v^{\beta}(\boldsymbol{x}+\boldsymbol{r})\right\rangle$ is proprtional to $r$ and hence $S_{(\ell=0)}^{\alpha \beta}(\boldsymbol{r}) \sim r^{2-\xi}$.

Returning to the main question of this paper, we see that no saturation of the anisotropic exponents occurs since the leading exponent in every $\ell>2$ sector is $\zeta^{(\ell)} \simeq \ell-2$. These exponents are within the window of locality of Eq. (5.2), which is given by $-\ell-3<\zeta<\ell-\xi$. However, the next-to-leading exponents (that are the leading ones in the structure function for $\ell=0,2$ ) are already out of this window and their relevance has to be discussed. We propose that the same mechanism that works in the toy model (see sec. IVB) also operates here and that all these higher exponents can be found in the full solution. To understand this, let us write a model equation for the correlation function in the spirit of Eq.(1.1),

$$
\hat{\mathcal{D}} C(\boldsymbol{r})+\int d \boldsymbol{y} K(\boldsymbol{r}-\boldsymbol{y}) C(\boldsymbol{y})=F(\boldsymbol{r})
$$


with $K$ being some kernel, and $\hat{\mathcal{D}}$ being some local differential operator. In view of Eq. (5.2), the differential operator $\hat{\mathcal{D}}$ should be regarded as the Kraichnan operator and the integral term should be taken for all integral terms in the equation including integrals due to the projection operators. These integrals create a window of locality that we denote by $\lambda_{\text {low }}<\lambda<\lambda_{\text {hi }}$. Any pure scaling solution $C(\boldsymbol{r}) \sim r^{\lambda}$ with $\lambda$ outside the window of locality will diverge and hence will not solve the homogeneous part of Eq. (5.41). Nevertheless, we will now demonstrate how this zero mode can be a part of a full solution without breaking scale invariance. For this we act with a Laplacian on both sides of Eq. (5.41) in order to get rid of the projection operators integrals. Of course, like in the linear pressure model, this will not eliminate all integral terms, and thus we can write the resultant equation as

$$
\partial^{2} \hat{\mathcal{D}} C(\boldsymbol{r})+\int d \boldsymbol{y} K(\boldsymbol{r}-\boldsymbol{y}) \partial^{2} C(\boldsymbol{y})=\partial^{2} F(\boldsymbol{r}) .
$$

Our main assumption, which was proven analytically in the simple case of the toy model, is that the above equation has a solution that is finite for all $r$ and decays for $r \gg L$. Let us now consider the zero modes of Eq. (5.42); their exponents have to be within the "shifted" window of locality $\lambda_{\text {low }}+2<\lambda<\lambda_{\text {hi }}+2$. Suppose now that $r^{\lambda}$ with $\lambda_{\text {hi }}<\lambda<\lambda_{\text {hi }}+2$ is such a solution, which is therefore part of the full solution of Eq. (5.42). We now claim that this solution also solves the original equation [Eq. (5.41)], hence allowing the existence of scaling exponents outside its window of locality. To see that, we first notice that since the full solution decays for $r \gg L$, then all the integrals in Eq. (5.41) converge and are therefore well defined. All that is left to show is that the equation is indeed solved by $C(\boldsymbol{r})$. But this is a trivial consequence of the uniqueness of the solution for Laplace equation with zero at infinity boundary conditions. Indeed, if we denote the integral term in Eq. (5.41) by

$$
I(\boldsymbol{r})=\int d \boldsymbol{y} K(\boldsymbol{r}-\boldsymbol{y}) C(\boldsymbol{y}),
$$

then from Eq. (5.42) we have

$$
\partial^{2} I(\boldsymbol{r})=\partial^{2}[F(\boldsymbol{r})-\hat{\mathcal{D}} C(\boldsymbol{r})],
$$

and since both $I(\boldsymbol{r})$ and $F(\boldsymbol{r})-\hat{\mathcal{D}} C(\boldsymbol{r})$ decay as $r \rightarrow \infty$, then they must be equal. Of course no breaking of scale invariance occurs because the equation is satisfied and $F(\boldsymbol{r})-\hat{\mathcal{D}} C(\boldsymbol{r})$ is a sum of an inhomogeneous solution and power laws.

Returning to the linear pressure model, we have shown that not only the first, leading exponents in every sector are legitimate but also the next few exponents. These exponents are inside the shifted window of locality of the "Laplaced" equation (5.4), which is given by $-\ell+1<$ $\lambda<\ell+4-\xi$.

At this point, we may ask whether this is also the case for the other exponents, which are outside this shifted window of locality. In light of the above discussion, it is clear that all of them may also be part of the full solution for we can always differentiate Eq. (5.2) sufficient number of times, thus shifting the window of locality to include any of these exponents. However, this procedure is unnecessary once we have written the prefactor $A(\lambda ; \ell, \xi)$ as an infinite sum of poles in $\lambda$. In that case the equation is defined for all values of $\lambda$ except for a discrete set of poles, enabling us to look for exponents as high as we wish. 

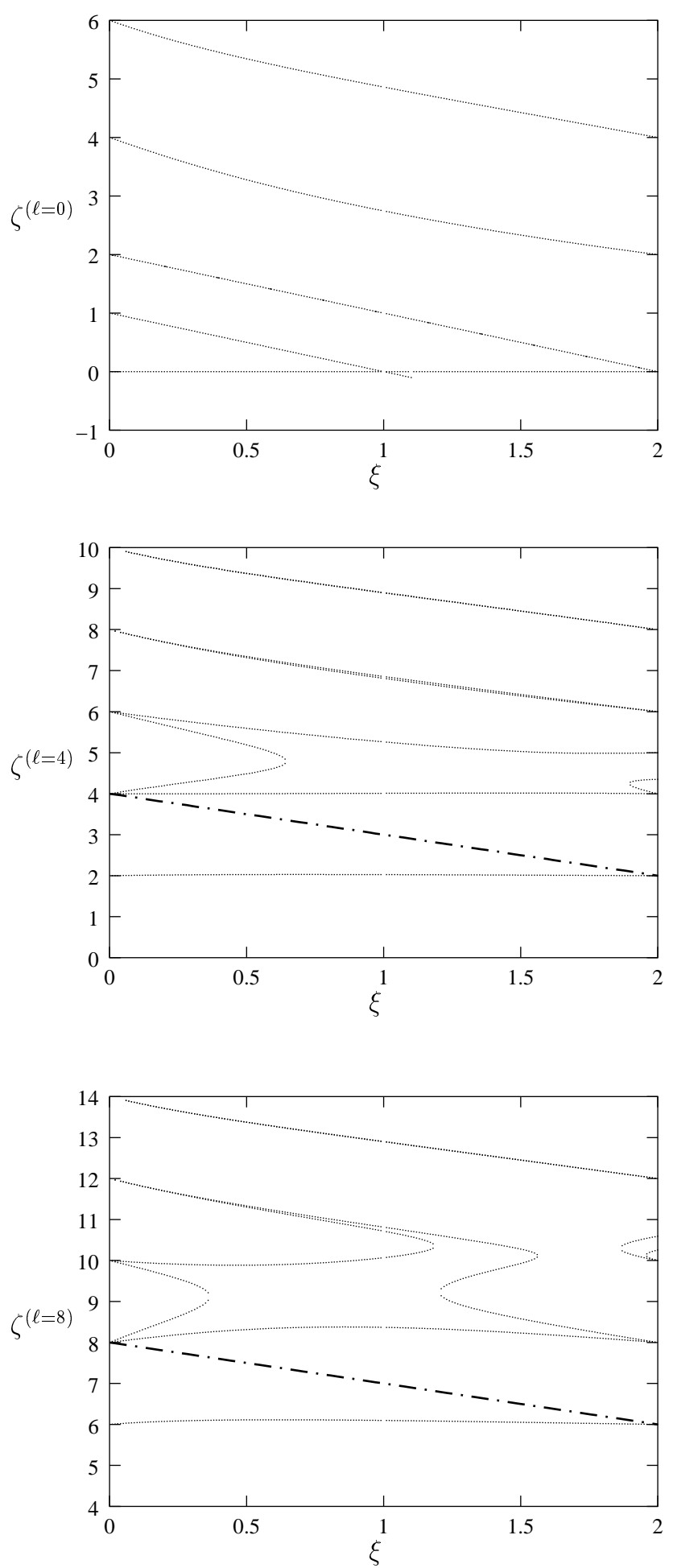

FIG. 2. Leading scaling exponents for the first few $\ell$ 's in the Linear Pressure model. The dashed line indicates the upper bound of the window of locality

\section{SUMMARY AND CONCLUSIONS}

1

The main question raised and answered in this paper is whether the existence of the pressure terms necessarily leads to a saturation of the scaling exponents associated with the anisotropic sectors. Such terms involve integrals
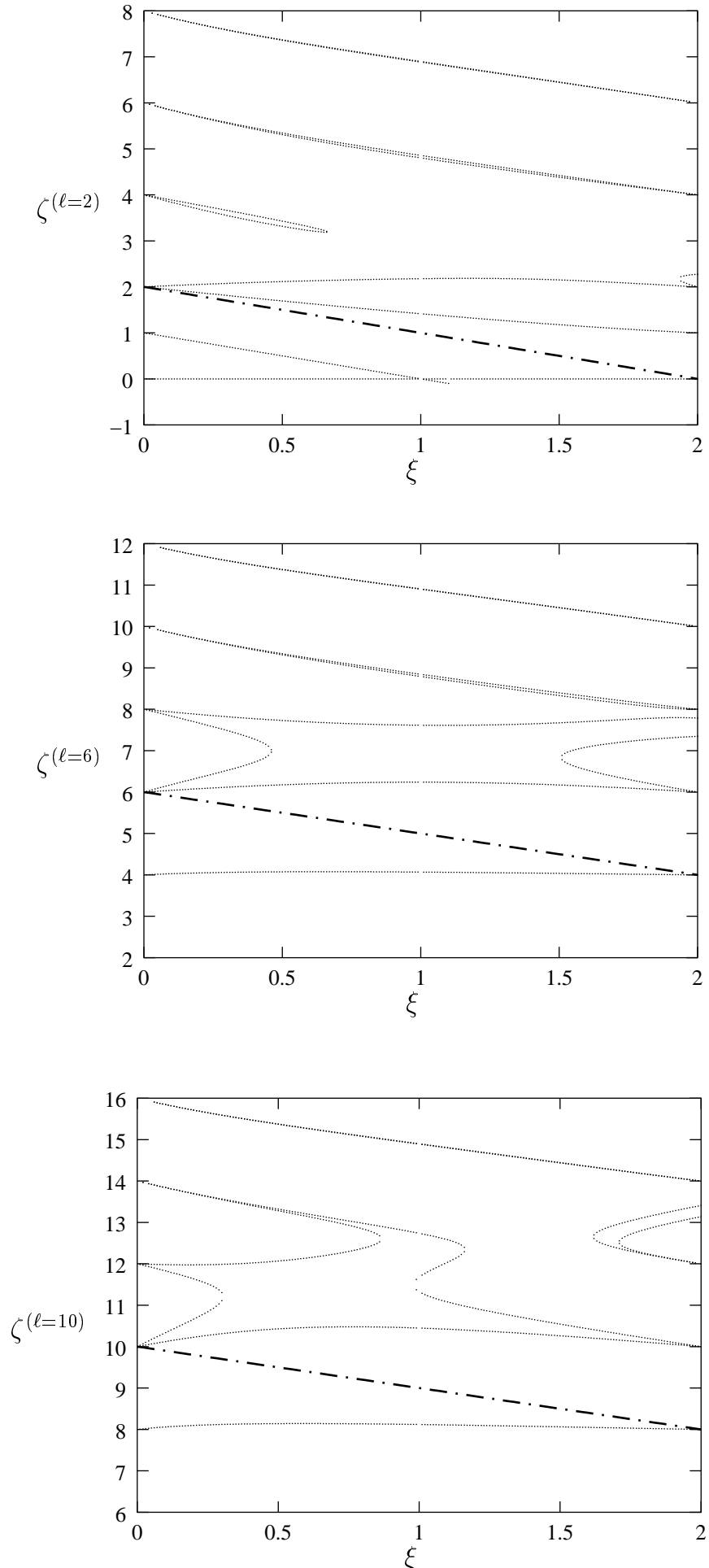

$$
\text { . }
$$


of the linear pressure model. The mechanism is based on two fundamental observations. The first one is that the window of locality widens up linearly in $\ell$ due to the angular integration. The second, and more important, is that a scaling solution with an unbounded spectrum can exist as a part of a full solution, which decays at infinity. Indeed pure scaling solutions cannot solve by themselves the zero-modes equation if their scaling exponent is out of the window of locality. However, the zero-modes are always part of the full solution that decays to zero once $r \gg L$ and we have shown that if such a solution solves a differential version of the full equation, it must also solve the original equation. Therefore by differentiating the full equation sufficiently many times, we can always reach a differential equation with a window of locality as high as we wish. In that equation we can find zero-mode solutions with arbitrarily high exponents (notice that in the toy model, it was sufficient to differentiate once to get rid of all integrals, thus obtaining an "infinitely wide" window of locality). But since these zero-modes are part of a full solution that decays at infinity, then this solution is also valid for the original equation, hence showing that in the full solution there can be power laws with arbitrarily high exponents.

Finally, we want to comment about the relevance of our calculations to Navier-Stokes turbulence. If we substitute blindly $\xi=4 / 3$ in our results, we predict the exponents $2 / 3,1.252$ 26, $2.01922,4.04843,6.06860$ and 8.08337 for $\ell=0,2,4,6,8$, and 10 , respectively. It would be tempting to propose that similar numbers may be expected for Navier-Stokes and indeed for $\ell=0$ and 2 this is not too far from the truth. We cannot, however, state with confidence that the genuine nonlinearity of Navier-Stokes does not change these numbers significantly. More work is needed before we can draw final conclusions on the rate of decay of the high sectors of anisotropy in Navier-Stokes turbulence.

\section{ACKNOWLEDGMENTS}

We thank Luca Biferale, Yoram Cohen and Massimo Vergassola for many helpful discussions and suggestions. We would also like to thank Anna Pomyalov for her help in the numerical calculations. This work has been supported in part by the Israel Science Foundation, the German-Israeli Foundation, the European Commission under contract No. HPRN-CT-2000-00162 ("Nonideal Turbulence"), and the Naftali and Anna BackenrothBronicki Fund for Research in Chaos and Complexity.

\section{APPENDIX A: GAUSSIAN INTEGRATION BY PARTS}

The field $\boldsymbol{w}(\boldsymbol{x}, t)$ as well as the forcing are Gaussian white noises. This enables us to express $T^{\alpha \beta}(\boldsymbol{r})$ and the correlation of the force in terms of $C^{\alpha \beta}(\boldsymbol{r})$ and $F^{\alpha \beta}(\boldsymbol{r})$. One way to accomplish this is by using the Gaussian integration by parts method [14]. Using the basic formula for Gaussian integration by parts, we get for the third moment

$$
\begin{gathered}
\left\langle v^{\alpha}(\boldsymbol{x}+\boldsymbol{r}, t) w^{\mu}(\boldsymbol{x}, t) v^{\beta}(\boldsymbol{x}, t)\right\rangle= \\
\int d t^{\prime} \int d \boldsymbol{y}\left\langle w^{\mu}(\boldsymbol{x}, t) w^{\nu}\left(\boldsymbol{y}, t^{\prime}\right)\right\rangle \\
\times\left[\left\langle\frac{\delta v^{\alpha}(\boldsymbol{x}+\boldsymbol{r}, t)}{\delta w^{\nu}\left(\boldsymbol{y}, t^{\prime}\right)} v^{\beta}(\boldsymbol{x}, t)\right\rangle\right. \\
\left.+\left\langle v^{\alpha}(\boldsymbol{x}+\boldsymbol{r}, t) \frac{\delta v^{\beta}(\boldsymbol{x}, t)}{\delta w^{\nu}\left(\boldsymbol{y}, t^{\prime}\right)}\right\rangle\right] .
\end{gathered}
$$

To find out the functional derivative, we formally integrate $v^{\alpha}(\boldsymbol{x}, t)$

$$
\begin{aligned}
& v^{\alpha}(\boldsymbol{x}, t)=\int_{-\infty}^{t} d t^{\prime} \partial_{t^{\prime}} v^{\alpha}\left(\boldsymbol{x}, t^{\prime}\right) \\
& =-\int_{-\infty}^{t} d t^{\prime} w\left(\boldsymbol{x}, t^{\prime}\right)^{\mu} \partial_{\mu} v^{\alpha}\left(\boldsymbol{x}, t^{\prime}\right) \\
& +\int_{-\infty}^{t} d t^{\prime} \int d \boldsymbol{y}\left[\partial^{\alpha} \partial_{\tau} G(\boldsymbol{x}-\boldsymbol{y})\right] w^{\mu}\left(\boldsymbol{y}, t^{\prime}\right) \partial_{\mu} v^{\tau}\left(\boldsymbol{y}, t^{\prime}\right) \\
& +[\text { terms that are independent of } \boldsymbol{w}]
\end{aligned}
$$

and thus

$$
\begin{aligned}
& \frac{\delta v^{\alpha}(\boldsymbol{x}, t)}{\delta w^{\nu}\left(\boldsymbol{y}, t^{\prime}\right)}=\theta\left(t-t^{\prime}\right)\left[-\delta^{3}(\boldsymbol{x}-\boldsymbol{y}) \partial_{\nu} v^{\alpha}\left(\boldsymbol{y}, t^{\prime}\right)\right. \\
& \left.\quad+\left[\partial^{\alpha} \partial_{\tau} G(\boldsymbol{x}-\boldsymbol{y})\right] \partial_{\nu} v^{\tau}\left(\boldsymbol{y}, t^{\prime}\right)\right] .
\end{aligned}
$$

When we plug this result back to Eq. (A1) we face the problem of evaluating the step function $\theta\left(t-t^{\prime}\right)$ at $t=t^{\prime}$ due to the delta correlation in time of $\boldsymbol{w}(\boldsymbol{r}, t)$. To solve this problem in a "physical" way [14, we approximate the delta function of the white noise with a sharp even function, perform the integral, and only then take the white noise limit. Doing so we obtain the formal result $\theta(0)=1 / 2$ stemming from the fact that we approximate a delta function with an even function. Finally, we remark that this derivation corresponds to the Stratonovich interpretation of the stochasic equation Eq. (2.1.).

Next, we perform the spatial integration, arriving at

$$
\begin{aligned}
& \left\langle v^{\alpha}(\boldsymbol{x}+\boldsymbol{r}, t) w^{\mu}(\boldsymbol{x}, t) v^{\beta}(\boldsymbol{x}, t)\right\rangle \\
= & -\frac{1}{2} K^{\mu \nu}(\boldsymbol{r}) \partial_{\nu} C^{\alpha \beta}(\boldsymbol{r})+\frac{1}{2} \partial_{(r)}^{\alpha} \\
& \times \int d \boldsymbol{y} G(\boldsymbol{r}-\boldsymbol{y}) \partial_{\tau}\left[K^{\mu \nu}(\boldsymbol{y}) \partial_{\nu} C^{\tau \beta}(\boldsymbol{y})\right] \\
& +\frac{1}{2} \int d \boldsymbol{y} \partial^{\beta} \partial_{\tau} G(\boldsymbol{y})\left[K^{\mu \nu}(\boldsymbol{y}) \partial_{\nu}^{(y)} C^{\alpha \tau}(\boldsymbol{r}-\boldsymbol{y})\right],
\end{aligned}
$$

and therefore 


$$
\begin{aligned}
& T^{\alpha \beta}(\boldsymbol{r})=\partial_{\mu}^{(r)}\left\langle v^{\alpha}(\boldsymbol{x}+\boldsymbol{r}) w^{\mu}(\boldsymbol{x}) v^{\beta}(\boldsymbol{x})\right\rangle \\
& \quad=-\frac{1}{2} K^{\mu \nu}(\boldsymbol{r}) \partial_{\mu} \partial_{\nu} C^{\alpha \beta}(\boldsymbol{r}) \\
& \quad+\frac{1}{2} \partial_{(r)}^{\alpha} \int d \boldsymbol{y} G(\boldsymbol{r}-\boldsymbol{y}) \partial_{\tau}\left[K^{\mu \nu}(\boldsymbol{y}) \partial_{\mu} \partial_{\nu} C^{\tau \beta}(\boldsymbol{y})\right] \\
& \quad-\frac{1}{2} \int d \boldsymbol{y} \partial^{\beta} \partial_{\tau} G(\boldsymbol{y})\left[K^{\mu \nu}(\boldsymbol{y}) \partial_{\nu}^{(y)} \partial_{\mu}^{(y)} C^{\alpha \tau}(\boldsymbol{r}-\boldsymbol{y})\right]
\end{aligned}
$$

\section{APPENDIX B: SIMPLIFICATION OF THE "NONLOCAL" TERM}

For $\xi \neq 1$, the nontrivial integral on the LHS of Eq. (2.25) can be further simplified. To see that, let us denote it by $I_{n t}$ and rewrite it (omitting the $-1 / 2$ factor) with the explicit forms of the Kraichnan operator and of the Green function

$$
\begin{aligned}
I_{n t} & =-(\xi+2) \frac{D}{4 \pi} \int d \boldsymbol{y} \frac{1}{y} \partial^{\beta} \partial_{\tau}\left[y^{\xi} \partial^{2} C^{\alpha \tau}(\boldsymbol{r}-\boldsymbol{y})\right] \\
& +\xi \frac{D}{4 \pi} \int d \boldsymbol{y} \frac{1}{y} \partial^{\beta} \partial_{\tau}\left[y^{\xi-2} y^{\mu} y^{\nu} \partial_{\mu} \partial_{\nu} C^{\alpha \tau}(\boldsymbol{r}-\boldsymbol{y})\right] .
\end{aligned}
$$

It is easy to verify that the tensors $\partial^{2} C^{\alpha \tau}(\boldsymbol{r}-\boldsymbol{y})$ and $y^{\mu} y^{\nu} \partial_{\mu} \partial_{\nu} C^{\alpha \tau}(\boldsymbol{r}-\boldsymbol{y})$ are divergence-free in both indices due to the fact that $C^{\alpha \tau}(\boldsymbol{r})$ itself is divergence free. Therefore, to simplify the integrals in Eq. (B1), we consider the generic expression

$$
\int d \boldsymbol{y} \frac{1}{y} \partial^{\beta} \partial_{\tau} y^{\lambda} X^{\alpha \tau}(\boldsymbol{y})
$$

where $X^{\alpha \tau}(\boldsymbol{y})$ is some divergence-free tensor and $\lambda$ is an arbitrary exponent. We also assume that $\lambda$ is such that the integral is convergent and integration by parts is allowed. Then we may write

$$
\begin{aligned}
& \int d \boldsymbol{y} \frac{1}{y} \partial^{\beta} \partial_{\tau} y^{\lambda} X^{\alpha \tau}(\boldsymbol{y}) \\
& =\int d \boldsymbol{y}\left[\lambda(\lambda-2) y^{\lambda-5} y^{\beta} y_{\tau} X^{\alpha \tau}(\boldsymbol{y})\right. \\
& \left.\quad+\lambda y^{\lambda-3} X^{\alpha \beta}(\boldsymbol{y})+\lambda y^{\lambda-3} y_{\tau} \partial^{\beta} X^{\alpha \tau}(\boldsymbol{y})\right] .
\end{aligned}
$$

The last formula can be simplified by using identity

$$
\begin{aligned}
0= & \int d \boldsymbol{y} \partial^{\beta} y^{\lambda-3} y_{\tau} X^{\alpha \tau}(\boldsymbol{y}) \\
= & (\lambda-3) \int d \boldsymbol{y} y^{\lambda-5} y^{\beta} y_{\tau} X^{\alpha \tau}(\boldsymbol{y}) \\
& +\int d \boldsymbol{y} y^{\lambda-3} X^{\alpha \beta}(\boldsymbol{y})+\int d \boldsymbol{y} y^{\lambda-3} y_{\tau} \partial^{\beta} X^{\alpha \tau}(\boldsymbol{y}),
\end{aligned}
$$

from which we get that for $\lambda \neq 3$

$$
\begin{aligned}
& \int d \boldsymbol{y} \frac{1}{y} \partial^{\beta} \partial_{\tau} y^{\lambda} X^{\alpha \tau}(\boldsymbol{y}) \\
& =-\frac{\lambda}{\lambda-3} \int d \boldsymbol{y}\left[y^{\lambda-3} X^{\alpha \beta}(\boldsymbol{y})+y^{\lambda-3} y_{\tau} \partial^{\beta} X^{\alpha \tau}(\boldsymbol{y})\right] .
\end{aligned}
$$

For $\lambda \neq 1$, we can even do better using the identity

$$
\begin{aligned}
0 & =\int d \boldsymbol{y} \partial_{\tau} y^{\lambda-1} \partial^{\beta} X^{\alpha \tau}(\boldsymbol{y}) \\
& =(\lambda-1) \int d \boldsymbol{y} y^{\lambda-3} y_{\tau} \partial^{\beta} X^{\alpha \tau}(\boldsymbol{y}),
\end{aligned}
$$

which finally brings us to

$$
\int d \boldsymbol{y} \frac{1}{y} \partial^{\beta} \partial_{\tau} y^{\lambda} X^{\alpha \tau}(\boldsymbol{y})=-\frac{\lambda}{\lambda-3} \int d \boldsymbol{y} y^{\lambda-3} X^{\alpha \beta}(\boldsymbol{y}) .
$$

Let us now apply Eq. (B8) to the integrals in Eq. (B1). Assuming that $\xi \neq 1$, we get

$$
\begin{aligned}
I_{n t}= & \frac{D \xi(\xi+2)}{4 \pi(\xi-3)} \int d \boldsymbol{y} y^{\xi-3} \partial^{2} C^{\alpha \beta}(\boldsymbol{r}-\boldsymbol{y}) \\
& -\frac{D \xi(\xi-2)}{4 \pi(\xi-5)} \int d \boldsymbol{y} y^{\xi-5} y^{\mu} y^{\nu} \partial_{\mu} \partial_{\nu} C^{\alpha \beta}(\boldsymbol{r}-\boldsymbol{y}) .
\end{aligned}
$$

To continue, we wish to turn the second integral into the same form of the first integral. To accomplish that, consider the following identity:

$$
\begin{aligned}
0= & \int d \boldsymbol{y} \partial^{\mu}\left[y^{\xi-3} y^{\nu} \partial_{\mu} \partial_{\nu} C^{\alpha \beta}(\boldsymbol{r}-\boldsymbol{y})\right] \\
= & (\xi-3) \int d \boldsymbol{y} y^{\xi-5} y^{\mu} y^{\nu} \partial_{\mu} \partial_{\nu} C^{\alpha \beta}(\boldsymbol{r}-\boldsymbol{y}) \\
& +\int d \boldsymbol{y} y^{\xi-3} \partial^{2} C^{\alpha \beta}(\boldsymbol{r}-\boldsymbol{y}) \\
& +\int d \boldsymbol{y} y^{\xi-3} y^{\nu} \partial_{\nu} \partial^{2} C^{\alpha \beta}(\boldsymbol{r}-\boldsymbol{y}),
\end{aligned}
$$

which gives us

$$
\begin{aligned}
& \int d \boldsymbol{y} y^{\xi-5} y^{\mu} y^{\nu} \partial_{\mu} \partial_{\nu} C^{\alpha \tau}(\boldsymbol{r}-\boldsymbol{y}) \\
& =-\frac{1}{\xi-3} \int d \boldsymbol{y}\left[y^{\xi-3} \partial^{2} C^{\alpha \beta}(\boldsymbol{r}-\boldsymbol{y})\right. \\
& \left.+y^{\xi-3} y^{\nu} \partial_{\nu} \partial^{2} C^{\alpha \beta}(\boldsymbol{r}-\boldsymbol{y})\right] .
\end{aligned}
$$

Additionally, we have

$$
\begin{aligned}
0 & =\int d \boldsymbol{y} \partial_{\nu} y^{\xi-3} y^{\nu} \partial^{2} C^{\alpha \beta}(\boldsymbol{y}) \\
& =\xi \int d \boldsymbol{y} y^{\xi-3} \partial^{2} C^{\alpha \beta}(\boldsymbol{y})+\int d \boldsymbol{y} y^{\xi-3} y^{\nu} \partial_{\nu} \partial^{2} C^{\alpha \beta}(\boldsymbol{y})
\end{aligned}
$$

and so finally we obtain

$$
\begin{aligned}
& \int d \boldsymbol{y} y^{\xi-5} y^{\mu} y^{\nu} \partial_{\mu} \partial_{\nu} C^{\alpha \tau}(\boldsymbol{r}-\boldsymbol{y}) \\
& =\frac{\xi-1}{\xi-3} \int d \boldsymbol{y} y^{\xi-3} \partial^{2} C^{\alpha \beta}(\boldsymbol{r}-\boldsymbol{y}) .
\end{aligned}
$$

Substituting this into Eq. (B9), we arrive at the final result for $I_{n t}$ :

$$
I_{n t}=\frac{12 \xi D}{(\xi-3)(\xi-5)} \int d \boldsymbol{y} G(\boldsymbol{y}) y^{\xi-2} \partial^{2} C^{\alpha \beta}(\boldsymbol{r}-\boldsymbol{y})
$$




\section{APPENDIX C: CALCULATION OF $A(\lambda ; \ell, \xi)$}

The prefactor $A(\lambda ; \ell, \xi)$ was defined by

$$
\begin{aligned}
& A(\lambda ; \ell, \xi) r^{\lambda+\xi} Y_{\ell m}(\hat{\boldsymbol{r}}) \\
& \quad \equiv \int d \boldsymbol{y} G(\boldsymbol{r}-\boldsymbol{y})|\boldsymbol{r}-\boldsymbol{y}|^{\xi-2} y^{\lambda} Y_{\ell m}(\hat{\boldsymbol{y}}) .
\end{aligned}
$$

Due to the isotropy of the integral, it is $m$ independent and therefore we specialize to the $m=0$ where the $Y_{\ell m}(\hat{\boldsymbol{y}})$ is proportional to the Legendre polynomial $P_{\ell}(\hat{\boldsymbol{y}} \cdot \hat{\boldsymbol{z}})$. Setting $\boldsymbol{r}=\hat{\boldsymbol{z}}$, the unit vector in the $z$ direction, we write the integral in spherical variables $(y, \theta, \phi)$ and perform the trivial $\phi$ integration (for $\boldsymbol{r}=\hat{\boldsymbol{z}}$, the integrand is independent of $\phi)$. We arrive at

$$
\begin{aligned}
A(\lambda ; \ell, \xi)= & -\frac{1}{2} \int_{0}^{\infty} d y y^{2+\lambda} \int_{-1}^{1} d(\cos \theta) \\
& \times\left(y^{2}-2 y \cos \theta+1\right)^{\frac{\xi-3}{2}} P_{\ell}(\cos \theta) .
\end{aligned}
$$

Using the standrad tricks of Feynmann integrals, one can express this integral (at least in the $\ell=0$ case) in terms of gamma functions. Here, however, we choose to calculate this integral directly by a strightforward expansion of the integrand. This procedure underlines the connection between the pole structure and the anisotropy label $\ell$.

Let us therefore turn the annoying $\left(y^{2}-2 y \cos \theta+\right.$ $1)^{(\xi-3) / 2}$ term into a Taylor series in $2 y \cos \theta /\left(1+y^{2}\right)$,

$$
\begin{aligned}
& A(\lambda ; \ell, \xi)=-\frac{1}{2} \sum_{n=0}^{\infty} \frac{(-1)^{n} 2^{n}}{n !} \\
& \quad \times\left(\frac{\xi-3}{2}\right) \cdot\left(\frac{\xi-3}{2}-1\right) \cdots\left(\frac{\xi-3}{2}-n+1\right) \\
& \quad \times B(n, \ell) C(\lambda, n, \xi),
\end{aligned}
$$

where $B(\lambda, n, \xi)$ and $C(n, \ell)$ are two one-dimensional integrals that are given by

$$
\begin{gathered}
B(n, \ell) \equiv \int_{-1}^{1} d(\cos \theta) \cos ^{n} \theta P_{\ell}(\cos \theta), \\
C(\lambda, n, \xi) \equiv \int_{0}^{\infty} d y y^{2+n+\lambda}\left(1+y^{2}\right)^{\frac{\xi-3}{2}-n} .
\end{gathered}
$$

Before calculating $B(n, \ell)$, we notice that it vanishes for $n<\ell$ and for $n$ s that are of different parity than $\ell$. The first observation is a simple manifestation of the orthogonality of the Legendre polynomials intimately connected to the orthogonality of different irreducible representations of the $\mathrm{SO}(3)$ group. For $n>\ell$ with the same parity, we can use the well-known identity

$$
P_{\ell}(x)=\frac{1}{2^{\ell} \ell !} \frac{d^{\ell}}{d x^{\ell}}\left(x^{2}-1\right)^{\ell},
$$

from which we get, after simple integration by parts, that $B(n, \ell)$ is given by

$$
\begin{aligned}
B(n, \ell)= & (-1)^{\ell} \frac{n(n-1) \cdots(n-\ell+1)}{2^{\ell} \ell !} \\
& \times \int_{-1}^{1} d x x^{n-\ell}\left(x^{2}-1\right)^{\ell} d x
\end{aligned}
$$

The above integral can be done explicitly leading us to the final result

$$
\begin{aligned}
B(n, \ell)= & \frac{n(n-1) \cdots(n-\ell+1)}{2^{\ell} \ell !} \\
& \times \sum_{k=0}^{n}\left(\begin{array}{l}
\ell \\
k
\end{array}\right) \frac{2(-1)^{k}}{n+2 k-\ell} .
\end{aligned}
$$

We now calculate the second integral by dividing the integration regime $[0,+\infty]$ in Eq. (C5) into a $0<y<1$ part and a $1<y<\infty$ part. In each part we expand the $\left(1+y^{2}\right)^{(\xi-3) / 2-n}$ term in $y$ and $1 / y$, respectively, and perform the integration. After adding up these terms again, we arrive at the following sum

$$
\begin{aligned}
& C(\lambda, n, \xi)=\sum_{k=0}^{\infty} \frac{1}{k !}\left(\frac{\xi-3}{2}-n\right) \\
& \quad \times\left(\frac{\xi-3}{2}-n-1\right) \cdot \ldots \cdot\left(\frac{\xi-3}{2}-n-k+1\right) \\
& \quad \times\left[\frac{1}{\lambda+3+n+2 k}-\frac{1}{\lambda+\xi-n-2 k}\right] .
\end{aligned}
$$

Plugging these results back in Eq. (C3), we get

$$
\begin{aligned}
& A(\lambda ; \ell, \xi)=-\frac{(-1)^{\ell}}{2} \sum_{n=\ell, \ell+2, \ldots}^{\infty} \sum_{k=0}^{\infty} \frac{2^{n}}{n ! k !} \\
& \quad \times\left(\frac{\xi-3}{2}\right) \cdots\left(\frac{\xi-3}{2}-n-k+1\right) \\
& \quad \times\left[\frac{1}{\lambda+3+n+2 k}-\frac{1}{\lambda+\xi-n-2 k}\right] B(n, \ell) .
\end{aligned}
$$

To expose the structure of the poles in the above formula, it is convenient to change the order of summation by defining the new indices $q, j$,

$$
\begin{aligned}
& q \equiv \frac{n-\ell}{2}+k=0,1,2, \ldots, \\
& j \equiv \frac{n-\ell}{2}=0,1,2, \ldots, q
\end{aligned}
$$

and obtain

$$
\begin{aligned}
& A(\lambda ; \ell, \xi)=-\frac{(-1)^{\ell}}{2} \sum_{q=0}^{\infty} a_{q}(\ell, \xi) \\
& \quad \times\left[\frac{1}{\lambda+3+\ell+2 q}-\frac{1}{\lambda+\xi-\ell-2 q}\right],
\end{aligned}
$$

where $a_{q}(\ell, \xi)$ are given by 


$$
\begin{aligned}
& a_{q}(\ell, \xi) \equiv \sum_{j=0}^{q} \frac{2^{\ell+2 j}}{(\ell+2 j) !(q-j) !} \quad(\mathrm{C} 13) \\
& \quad \times\left(\frac{\xi-3}{2}\right) \cdot \ldots \cdot\left(\frac{\xi-3}{2}-\ell-j-q+1\right) B(\ell+2 j, \ell) .
\end{aligned}
$$

Equation $\mathrm{C} 12$ shows that $A(\lambda ; \ell, \xi)$ is given as an infinite series of poles in $\lambda$. For $\xi>0$ this series can be shown to converge although for small values of $\xi$ the convergence is very slow. In the special case of $\xi=2$, the series is truncated after the first pole. To see why this is so, return to the original definition of $A(\lambda ; \ell, \xi)$ and set $\xi=2$ :

$$
A(\lambda ; \ell, 2) r^{\lambda+2} Y_{\ell m}(\hat{\boldsymbol{r}})=\int d \boldsymbol{y} G(\boldsymbol{r}-\boldsymbol{y}) y^{\lambda} Y_{\ell m}(\hat{\boldsymbol{y}}) .
$$

But since

$$
y^{\lambda} Y_{\ell m}(\hat{\boldsymbol{y}})=\frac{1}{(\lambda+2-\ell)(\lambda+3+\ell)} \partial^{2} y^{\lambda+2} Y_{\ell m}(\hat{\boldsymbol{y}})
$$

then from the definition of the Green function we get

$$
A(\lambda ; \ell, 2)=\frac{1}{(\lambda+2-\ell)(\lambda+3+\ell)} .
$$

[1] I. Arad, V. S. L'vov and I. Procaccia, Phys. Rev. E59, 6753 (1999).

[2] I. Arad, B. Dhruva, S. Kurien, V.S. L'vov, I. Procaccia and K.R.Sreenivasan, Phys. Rev. Lett., 81, 5330 (1998).

[3] I. Arad, L. Biferale, I. Mazzitelli and I. Procaccia, Phys. Rev. Lett. 82, 5040 (1999).

[4] S. Kurien, V. S. L'vov, I. Procaccia and K.R. Sreenivasan, Phys. Rev. E, 61, 407 (2000).

[5] R.H. Kraichnan, Phys. Fluids 11, 945 (1968)

[6] M. Vergassola, Phys. Rev. E 53, R3021 (1996).

[7] I. Arad, V. S. L'vov, E. Podivilov and I. Procaccia, Phys Rev. E, 62, 4904 (2000)

[8] I. Arad, L. Biferale and I. Procaccia, Phys.Rev.E, 61, 2654 (2000)

[9] V. L'vov and I. Procaccia, Phys. Rev. E, 52, 3840 (1995).

[10] K. Gawedzki and A. Kupiainen, Phys. Rev. Lett. 75, 3834 (1995)

[11] M. Chertkov, G. Falkovich, I. Kolokolov and V. Lebedev, Phys. Rev E 52, 4924 (1995).

[12] D. Fukayama, T. Oyamada, T. Nakano, T. Gotoh and K. Yamamoto, J. Phys. Soc. Japan 69, 701, 2000

[13] U. Frisch, Turbulence, the Legacy of A. N. Kolmogorov (Cambridge University Press, Cambridge, 1995).

[14] See, for example, J. Zinn-Justin, Quantum field Theory and Critical Phenomena, 2nd ed. (Clarendon Press, Oxford, 1993), Chap. 4. 